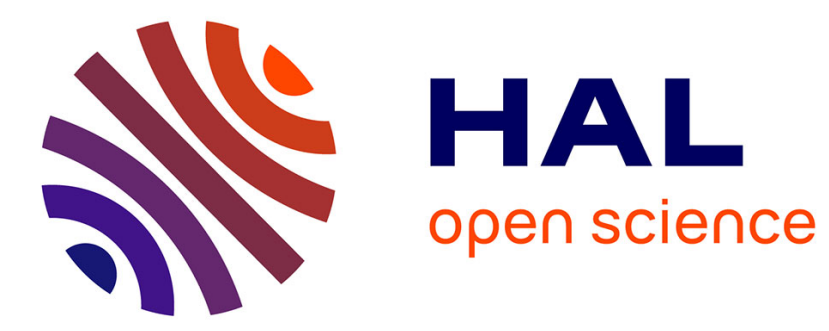

\title{
Existence result for the coupling problem of two scalar conservation laws with Riemann initial data
}

Benjamin Boutin, Christophe Chalons, Pierre-Arnaud Raviart

\section{To cite this version:}

Benjamin Boutin, Christophe Chalons, Pierre-Arnaud Raviart. Existence result for the coupling problem of two scalar conservation laws with Riemann initial data. Mathematical Models and Methods in Applied Sciences, 2010, 20 (10), pp.1859-1898. 10.1142/S0218202510004817 . hal-00871839

\section{HAL Id: hal-00871839 \\ https://hal.science/hal-00871839}

Submitted on 10 Oct 2013

HAL is a multi-disciplinary open access archive for the deposit and dissemination of scientific research documents, whether they are published or not. The documents may come from teaching and research institutions in France or abroad, or from public or private research centers.
L'archive ouverte pluridisciplinaire HAL, est destinée au dépôt et à la diffusion de documents scientifiques de niveau recherche, publiés ou non, émanant des établissements d'enseignement et de recherche français ou étrangers, des laboratoires publics ou privés. 


\title{
Existence result for the coupling problem of two scalar conservation laws with Riemann initial data
}

\author{
Benjamin Boutin Christophe Chalons \\ Pierre-Arnaud Raviart
}

January 6, 2010

\begin{abstract}
This paper is devoted to the coupling problem of two scalar conservation laws through a fixed interface located for instance at $\mathrm{x}=0$. Each scalar conservation law is associated with its own (smooth) flux function and is posed on a half-space, namely $x<0$ or $x>0$. At interface $x=0$ we impose a coupling condition whose objective is to enforce in a weak sense the continuity of a prescribed variable, which may differ from the conservative unknown (and the flux functions as well). We prove existence of a solution to the coupled Riemann problem using a constructive approach. The latter allows in particular to highlight interesting features like non uniqueness of both continuous and discontinuous (at interface $x=0$ ) solutions. The behavior of some numerical scheme is also investigated.
\end{abstract}

\section{Introduction}

The coupling of partial differential equations is of increasing interest in the applied mathematics community, and of course of increasing importance for industrial applications. Such a coupling arises for instance in the simulation of nuclear reactors when different two-phase flow codes are used ${ }^{1}$. In these codes, multiple modelling scales are applied to describe the flow. For instance, different thermal-hydraulic models can be used for each reactor component to take into account its specific behavior, or small scale models can be used, locally, to obtain a better resolution. When these models are put side to side, we face the problem of coupling. In addition to the definition of each model, such a problematic requires to be supplemented with an interfacial model in order to precise the nature of the information that must be exchanged at the coupling interface. This interfacial model may be formulated for instance when imposing the continuity of a given set of variables. It generally strongly affects the whole solution and must therefore be defined in order to achieve a physically coherent description of the whole operating device under consideration.

\footnotetext{
${ }^{1}$ The authors of the present paper are involved in a joint research program on multiphase flows between CEA (French center fo nuclear research) and University Pierre et Marie CurieParis6 (Laboratoire Jacques-Louis Lions) in the frame of the Neptune project [20]. See for instance $[3,4,5,6,2,11]$ and the references therein
} 
Let us mention that similar situations appear in the modelling of networks and traffic flows which have received a certain interest in the last few years. We refer for instance the reader to $[12,8,9],[16,17,21]$, and the references therein.

In this paper, we are interested in the one dimensional coupling problem of two scalar conservation laws through a fixed interface, say $x=0$, and more precisely in the resolution of the coupled Riemann problem. Each scalar conservation law is associated with its own (smooth) flux function $f_{\alpha}, \alpha=L, R$ and is posed on a half-space, namely $x<0(\alpha=L)$ or $x>0(\alpha=R)$. Note from now on that it will be implicitly assumed throughout the paper that the flux functions have at most a finite number of changes of convexity, which is often (is not always) the case for practical applications. At the coupling interface, we assume without further details that it is physically relevant to impose the continuity of a given function $v_{\alpha}=v_{\alpha}(u)$ of the solution $u$, meaning that $u$ is expected to satisfy

$$
v_{\alpha}\left(u\left(0_{-}, t\right)\right)=v_{\alpha}\left(u\left(0_{+}, t\right)\right), t>0 .
$$

Note that the $v$-variable generally depends on $\alpha$.

This approach is fairly general and referred to as the state coupling method. The theoretical study of such coupling conditions was initiated in the pioneering papers $[19,18]$ in the case of scalar equations, linear systems, and the usual Lagrangian system as well. In particular, the continuity constraint (1) has been reformulated in a weak sense inspired by [13] for the sake of well-posedness. This results in considering two boundary value problems and imposing "as far as possible" the continuity of the $v$-variable at the interface. We also refer the reader to $[5,11]$ and the references therein. It is important to notice that this approach does not ensure the conservativity property of the coupling problem. It does therefore significantly differ from the flux coupling method where the continuity of the flux is imposed at the interface $\left(v_{\alpha}(u)=f_{\alpha}(u)\right)$. See for instance $[23,24,7]$ and $[22]$.

This paper gives the first result of global existence of a solution to the coupled Riemann problem in this context of state coupling, using a constructive approach. Except the smoothness hypothesis, no specific assumption is made on the flux functions $f_{\alpha}, \alpha=L, R$. It is worth noticing right now that the solution of the coupled Riemann problem can be either continuous or discontinuous in the $v$-variable at the coupling interface. In the first situation, the coupling condition (1) is satisfied in the classical sense, while in the other one, it is satisfied in a weak sense only (to be precised hereafter). In addition, the solution to the Riemann problem is shown to be not necessarily unique, since in particular, a 1parameter family of continuous solutions at the coupling interface may exist for the same Riemann initial data. In this context, it is an open problem to know wether or not there exists any natural criterion based for instance on entropy or stability arguments for choosing one particular solution. Then, our approach is different from the ones adopted for instance in [1] or [15] where the authors give and study different concepts of physically admissible entropy weak solutions. So as to get for instance existence and uniqueness of the corresponding Riemann weak solutions.

The outline of the paper is as follows. In Section 1, we introduce the general framework of the state coupling method. Section 2 is devoted to the main result of this paper, namely the existence of a solution to the coupled Riemann problem. First of all, a geometrical description of the sets of admissible traces at the 
interface is given. Then, a characterisation is given for the solutions satisfying the coupling condition either in the strong sense (the so-called $v$-continuous solutions) or in the weak sense (the so-called $v$-discontinuous solutions). At last, we deduce the existence of at least one self-similar solution to any coupled Riemann problem. Several situations of non-uniqueness are exhibited, and a first case of coupling of scalar conservation laws "with phase change" is treated theoretically. Section 3 is devoted to numerical simulations, using both a relaxation and a Godunov scheme as a building block for the derivation of the numerical strategy.

\section{The state coupling method}

Let $f_{\alpha}: \mathbb{R} \rightarrow \mathbb{R}, \alpha=L, R$, be two $C^{1}$ functions; given a function $u_{0}: \mathbb{R} \rightarrow \mathbb{R}$, we want to find a function $u:(x, t) \rightarrow u(x, t) \in \mathbb{R}$ solution of

$$
\begin{aligned}
& \frac{\partial u}{\partial t}+\frac{\partial}{\partial x} f_{L}(u)=0, x<0, t>0 \\
& \frac{\partial u}{\partial t}+\frac{\partial}{\partial x} f_{R}(u)=0, x>0, t>0
\end{aligned}
$$

and satisfying the initial condition

$$
u(x, 0)=u_{0}(x), x \in \mathbb{R}
$$

together with coupling constraints at $x=0$ that we now define. Let $\theta_{\alpha}: \mathbb{R} \rightarrow \mathbb{R}$, $\alpha=L, R$, be two strictly monotone $C^{1}$ functions; we require the function $u$ to satisfy "as far as possible" the continuity constraint

$$
\theta_{L}^{-1}\left(u\left(0_{-}, t\right)\right)=\theta_{R}^{-1}\left(u\left(0_{+}, t\right)\right), t>0 .
$$

Setting

$$
v(x, t)=\left\{\begin{array}{c}
\theta_{L}^{-1}(u(x, t)), x<0 \\
\theta_{R}^{-1}(u(x, t)), x>0,
\end{array}\right.
$$

this constraint must be understood in the weak sense, following [13], [19] and [18]

$$
\left\{\begin{aligned}
v\left(0_{-}, t\right) & \in \widetilde{\mathcal{O}}_{L}\left(v\left(0_{+}, t\right)\right) \\
v\left(0_{+}, t\right) & \in \widetilde{\mathcal{O}}_{R}\left(v\left(0_{-}, t\right)\right) .
\end{aligned}\right.
$$

Let us recall the definition of the sets $\widetilde{\mathcal{O}}_{L}\left(v_{d}\right)$ and $\widetilde{\mathcal{O}}_{R}\left(v_{g}\right)$. Denoting by $w_{\alpha}\left(\frac{x}{t} ; u_{g}, u_{d}\right)$ the self-similar solution of the Riemann problem

$$
\begin{aligned}
& \frac{\partial u}{\partial t}+\frac{\partial}{\partial x} f_{\alpha}(u)=0, x \in \mathbb{R}, t>0 \\
& u(x, 0)=\left\{\begin{array}{l}
u_{g}, x<0 \\
u_{d}, x>0
\end{array}\right.
\end{aligned}
$$

and setting

$$
z_{\alpha}\left(\frac{x}{t} ; v_{g}, v_{d}\right)=\theta_{\alpha}^{-1}\left(w_{\alpha}\left(\frac{x}{t} ; \theta_{\alpha}\left(v_{g}\right), \theta_{\alpha}\left(v_{d}\right)\right)\right)
$$


we have

$$
\left\{\begin{array}{l}
\widetilde{\mathcal{O}}_{L}\left(v_{d}\right)=\left\{z_{L}\left(0_{-} ; v, v_{d}\right) ; v \in \mathbb{R}\right\} \\
\widetilde{\mathcal{O}}_{R}\left(v_{g}\right)=\left\{z_{R}\left(0_{+} ; v_{g}, v\right) ; v \in \mathbb{R}\right\}
\end{array}\right.
$$

\section{Solving the coupled Riemann problem.}

We consider the coupled Riemann problem which corresponds to the initial condition

$$
u_{0}(x)=\left\{\begin{array}{l}
u_{g}, x<0 \\
u_{d}, x>0 .
\end{array}\right.
$$

We set

$$
v_{g}=\theta_{L}^{-1}\left(u_{g}\right), v_{d}=\theta_{R}^{-1}\left(u_{d}\right) .
$$

When the flux functions $f_{\alpha}, \alpha=L, R$ are strictly convex, we are able to exhibit all the solutions of this coupled Riemann problem (2)-(4),(7),(8). This is indeed the goal of this section. Recall that in the general case, we always assume for simplicity that the flux functions have at most a finite number of changes of convexity.

\subsection{Preliminaries.}

Let us first recall and derive some preliminary results. Given a function $f \in$ $C^{1}(\mathbb{R})$, we consider the scalar equation

$$
\frac{\partial u}{\partial t}+\frac{\partial}{\partial x} f(u)=0 .
$$

We denote by $w\left(\frac{x}{t} ; u_{g}, u_{d}\right)$ the solution of the Riemann problem for (9) corresponding to the initial condition (8). In the case of a general flux function $f, w\left(\frac{x}{t} ; u_{g}, u_{d}\right)$ consists of a composite wave composed of shock and rarefaction subwaves. It is constructed in the following way.

(i) For $u_{d}>u_{g}$, we introduce the lower convex envelope function $f_{c}$ of $f$ in the interval $\left[u_{g}, u_{d}\right]$. This interval is divided into rarefaction subintervals where the function $f$ is strictly convex (so that $f_{c}=f$ ) separated by shock subintervals where the function $f_{c}$ is affine (and the graph of $f$ is located above the corresponding chord). Then $w\left(\frac{x}{t} ; u_{g}, u_{d}\right)$ is made of a sequence of rarefaction waves in the rarefaction subintervals and shock waves in the shock subintervals. These waves are bordered on the left by the constant state $u_{g}$ and on the right by the constant state $u_{d}$. These constant states are the only constant states which appear in the solution of the Riemann problem.

(ii) For $u_{d}<u_{g}$, we introduce the upper concave envelope function $f^{c}$ of $f$ in the interval $\left[u_{d}, u_{g}\right]$. Again this interval is divided into rarefaction subintervals where the function $f$ is strictly concave (so that $f=f^{c}$ ) separated by shock intervals where $f^{c}$ is affine (and the graph of $f$ is located under the corresponding chord. Then, the solution of the Riemann problem then has the same structure as in the case (i).

We shall say that such a composite wave has a nonnegative (resp. nonpositive) speed if all of its subwaves (shocks or rarefactions) have nonnegative (resp. nonpositive) speeds. In order to characterize the composite waves $w\left(. ; u_{g}, u_{d}\right)$ whose speeds are nonnegative or nonpositive, we determine the minimal and 
maximal speeds $\sigma_{\min }$ and $\sigma_{\max }$ of such a composite wave. Denote by $I\left(u_{g}, u_{d}\right)$ the closed interval whose end points are $u_{g}$ and $u_{d}$. Then, we can state

Lemma 1 We have

$$
\sigma_{m i n}=\min _{u \in I\left(u_{g}, u_{d}\right)} \frac{f(u)-f\left(u_{g}\right)}{u-u_{g}}
$$

and

$$
\sigma_{\max }=\max _{u \in I\left(u_{g}, u_{d}\right)} \frac{f(u)-f\left(u_{d}\right)}{u-u_{d}}
$$

with the following convention

$$
\frac{f(u)-f\left(u_{a}\right)}{u-u_{a}}=f^{\prime}\left(u_{a}\right) \text { for } u=u_{a}, a=g, d .
$$

Proof. Let us check (10) for instance. We begin by observing that $\sigma_{\min }$ is the speed of the left boundary of the fan of the composite wave $w\left(. ; u_{g}, u_{d}\right)$. Assume first $u_{d}>u_{g}$. If the left subwave of $w\left(. ; u_{g}, u_{d}\right)$ is a shock that connects $u_{g}$ and a state $u_{1}$, its speed is

$$
\sigma_{\min }=\frac{f\left(u_{1}\right)-f\left(u_{g}\right)}{u_{1}-u_{g}}
$$

On the other hand, it is clear geometrically (cf. Fig. 1a) that we have for all $u \in\left(u_{g}, u_{d}\right]$

$$
\frac{f(u)-f\left(u_{g}\right)}{u-u_{g}}>\frac{f\left(u_{1}\right)-f\left(u_{g}\right)}{u_{1}-u_{g}} .
$$

Hence, using the convention (12), we obtain

$$
\sigma_{\text {min }}=\min _{u \in\left[u_{g}, u_{d}\right]} \frac{f(u)-f\left(u_{g}\right)}{u-u_{g}} .
$$

If this left subwave is a rarefaction, $\sigma_{\min }$ is the speed of the left side of the rarefaction fan which is given by

$$
\sigma_{\min }=f^{\prime}\left(u_{g}\right)
$$

Again, it is obvious geometrically (cf. Fig. 1b) that we have for all $u \in\left(u_{g}, u_{d}\right]$

$$
f^{\prime}\left(u_{g}\right)<\frac{f(u)-f\left(u_{g}\right)}{u-u_{g}} .
$$

Therefore (13) still holds.

Consider next the case where $u_{g}>u_{d}$. Using Fig. 2 and a fairly similar analysis, one can check that

$$
\sigma_{\min }=\min _{u \in\left[u_{d}, u_{g}\right]} \frac{f(u)-f\left(u_{g}\right)}{u-u_{g}} .
$$

This proves (10). The property (11) is established exactly in the same way.

As a consequence of Lemma 1 , we obtain that a (composite) wave $w\left(. ; u_{g}, u_{d}\right)$ has a nonnegative speed if and only if

$$
\min _{u \in I\left(u_{g}, u_{d}\right)} \frac{f(u)-f\left(u_{g}\right)}{u-u_{g}} \geq 0
$$



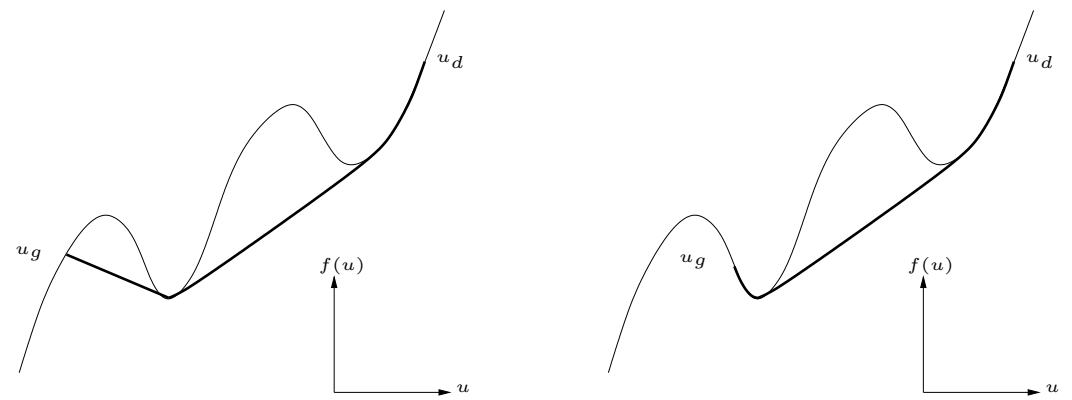

Figure 1: $u_{g}<u_{d}$ and the left subwave is a shock (a) or a rarefaction (b)
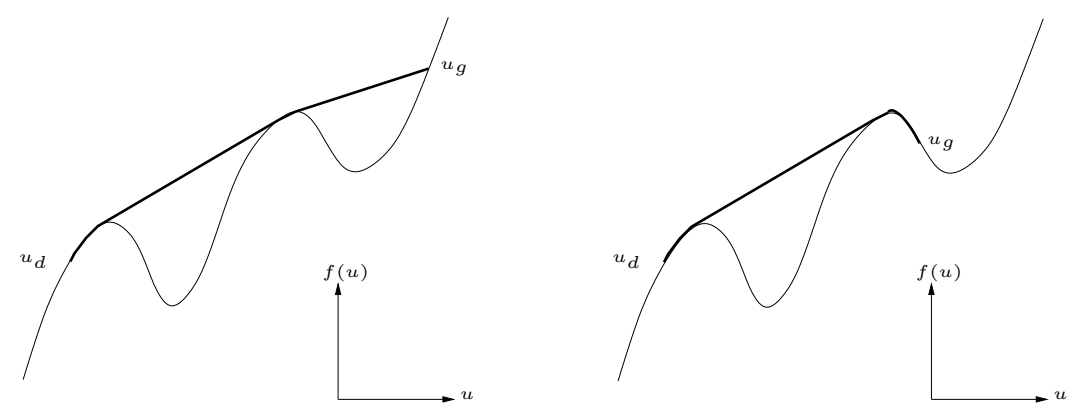

Figure 2: $u_{g}>u_{d}$ and the left subwave is a shock (a) or a rarefaction (b)

and a nonpositive speed if and only if

$$
\max _{u \in I\left(u_{g}, u_{d}\right)} \frac{f(u)-f\left(u_{d}\right)}{u-u_{d}} \leq 0 .
$$

In other words, we get

$$
\left\{\begin{array}{l}
w\left(0_{-} ; u_{g}, u_{d}\right)=u_{g} \Longleftrightarrow \min _{u \in I\left(u_{g}, u_{d}\right)} \frac{f(u)-f\left(u_{g}\right)}{u-u_{g}} \geq 0, \\
w\left(0_{+} ; u_{g}, u_{d}\right)=u_{d} \Longleftrightarrow \max _{u \in I\left(u_{g}, u_{d}\right)} \frac{f(u)-f\left(u_{d}\right)}{u-u_{d}} \leq 0 .
\end{array}\right.
$$

Now, given a state $u_{0}$, we look for the set $\mathcal{E}^{+}\left(u_{0}\right)$ (resp. $\mathcal{E}^{-}\left(u_{0}\right)$ ) of all states $u \neq u_{0}$ which can be connected to $u_{0}$ on the left (resp. on the right) by a nontrivial (composite) wave $w\left(\cdot ; u, u_{0}\right)$ (resp. $\left.w\left(\cdot ; u_{0}, u\right)\right)$ whose speed is nonnegative (resp. nonpositive). The above results yield

Lemma 2 We have

$$
\mathcal{E}^{+}\left(u_{0}\right)=\left\{u \neq u_{0} ; \min _{v \in I\left(u_{0}, u\right)} \frac{f(v)-f(u)}{v-u} \geq 0\right\}
$$

and

$$
\mathcal{E}^{-}\left(u_{0}\right)=\left\{u \neq u_{0} ; \max _{v \in I\left(u_{0}, u\right)} \frac{f(v)-f(u)}{v-u} \leq 0\right\}
$$


It remains to give a geometric characterization of the conditions (15) and (16). This is easily done when the flux function $f$ is either monotone or strictly convex.

Example 2.1. the case of a monotone flux function. If the function $f$ is strictly increasing so that

$$
\frac{f(v)-f(u)}{v-u}>0 \text { for all } v \neq u,
$$

we obtain

$$
\mathcal{E}^{+}\left(u_{0}\right)=\mathbb{R} \backslash\left\{u_{0}\right\}, \quad \mathcal{E}^{-}\left(u_{0}\right)=\emptyset
$$

while if the function $f$ is strictly decreasing, we find

$$
\mathcal{E}^{+}\left(u_{0}\right)=\emptyset, \quad \mathcal{E}^{-}\left(u_{0}\right)=\mathbb{R} \backslash\left\{u_{0}\right\} .
$$

Example 2.2. the case of a strictly convex flux function. When the function $f$ is strictly convex, we denote by $\bar{u}$ the sonic state of $f$ characterized by $f^{\prime}(\bar{u})=0$ with the convention that $\bar{u}=-\infty$ (resp. $\bar{u}=+\infty)$ if the function $f$ is strictly increasing (resp. strictly decreasing). With the state $u_{0}$, we associate the state $\tilde{u}_{0}$ defined by

$$
\left\{\begin{array}{l}
f\left(\widetilde{u}_{0}\right)=f\left(u_{0}\right), \tilde{u}_{0} \neq u_{0} \text { if } \bar{u} \text { exists } \\
\tilde{u}_{0}=\bar{u}=-\infty \quad \text { if } f \text { is strictly increasing, } \\
\tilde{u}_{0}=\bar{u}=+\infty \quad \text { if } f \text { is strictly decreasing. }
\end{array}\right.
$$

Lemma 3 Assume that the function $f$ is strictly convex. Then

$$
\mathcal{E}^{+}\left(u_{0}\right)=\left\{u \neq u_{0} ; u \geq \max \left(\bar{u}, \tilde{u}_{0}\right)\right\}
$$

and

$$
\mathcal{E}^{-}\left(u_{0}\right)=\left\{u \neq u_{0} ; u \leq \min \left(\bar{u}, \tilde{u}_{0}\right)\right\} .
$$

Proof. Let us check for instance the property (18). Given $u \in \mathbb{R}$, we define the function $g$ by

$$
g(v)=\frac{f(v)-f(u)}{v-u}, v \neq u, \quad g(u)=f^{\prime}(u) .
$$

Since

$$
g^{\prime}(v)=\frac{f(u)-f(v)+f^{\prime}(v)(v-u)}{(v-u)^{2}}
$$

and by the strict convexity of $f$

$$
f(u)-f(v)+f^{\prime}(v)(v-u)>0, \quad v \neq u,
$$

this function $g$ is strictly increasing. Hence we obtain

$$
\min _{v \in I\left(u_{0}, u\right)} g(v)=\left\{\begin{array}{l}
g\left(u_{0}\right)=\frac{f\left(u_{0}\right)-f(u)}{u_{0}-u} \quad \text { if } u_{0}<u \\
g(u)=f^{\prime}(u) \quad \text { if } u_{0}>u
\end{array}\right.
$$



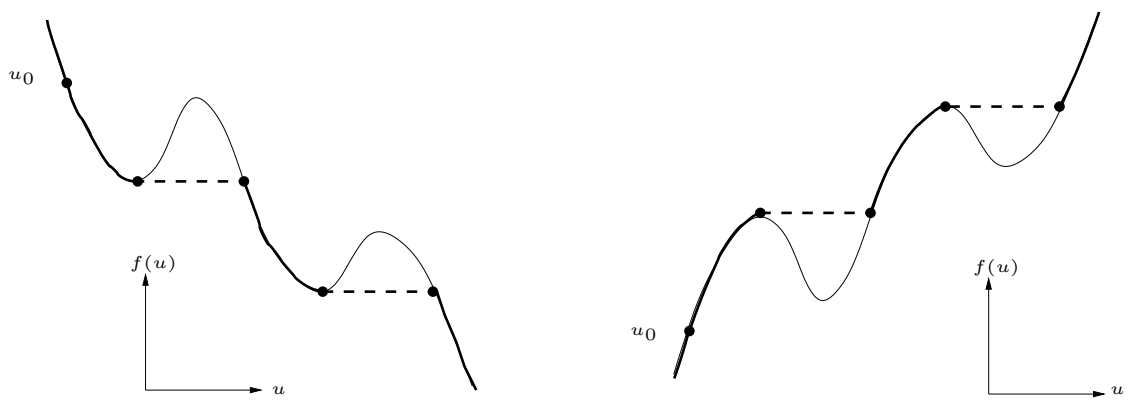

Figure 3: In bold-face, the sets $\mathcal{E}^{-}\left(u_{0}\right)$ (a) and $\mathcal{E}^{+}\left(u_{0}\right)$ (b), $u_{0}$ being excluded in both cases, and the other circle points being included

so that

$$
\min _{v \in I\left(u_{0}, u\right)} g(v) \geq 0 \Longleftrightarrow\left\{\begin{array}{l}
f(u) \geq f\left(u_{0}\right) \quad \text { if } u_{0}<u \\
f^{\prime}(u) \geq 0 \quad \text { if } u_{0}>u .
\end{array}\right.
$$

Now the condition $f(u) \geq f\left(u_{0}\right)$ for $u_{0}<u$ holds trivially if $u_{0} \geq \bar{u}$ but means $u \geq \tilde{u}_{0}$ if $u_{0} \leq \bar{u}$. On the other hand, the condition $f^{\prime}(u) \geq 0$ means $u \geq \bar{u}$. The property (18) is then proved.

In the above examples, $\mathcal{E}^{ \pm}\left(u_{0}\right)$ is an interval or the whole real line, the state $u_{0}$ being excluded. In the case of a general flux function, $\mathcal{E}^{ \pm}\left(u_{0}\right)$ consists of an interval or a union of disjoint intervals (cf. Fig. 3).

We next give another useful characterization of the sets $\mathcal{E}^{ \pm}\left(u_{0}\right)$

Lemma 4 We have

$$
\mathcal{E}^{+}\left(u_{0}\right)=\left\{u=w\left(0_{-} ; y, u_{0}\right), y \in \mathbb{R} ; u \neq u_{0}\right\}
$$

and

$$
\mathcal{E}^{-}\left(u_{0}\right)=\left\{u=w\left(0_{+} ; u_{0}, y\right), y \in \mathbb{R} ; u \neq u_{0}\right\} .
$$

Proof. Let $u=w\left(0_{-} ; y, u_{0}\right) \neq u_{0}$ for some $y \in \mathbb{R}$. Then, $u$ is connected to $u_{0}$ by a wave $w\left(\cdot ; u, u_{0}\right)$ whose speed is nonnegative, i.e., $u \in \mathcal{E}^{+}\left(u_{0}\right)$. Conversely, if $u \in \mathcal{E}^{+}\left(u_{0}\right)$, then $u=w\left(0_{-} ; u, u_{0}\right) \neq u_{0}$ which proves (20). The property (21) is established in a similar way.

In the sequel, we will make use of the following sets:

$$
\left\{\begin{array}{l}
\mathcal{F}^{+}\left(u_{0}\right)=\left\{u=w\left(0_{+} ; y, u_{0}\right), y \in \mathbb{R} ; u \neq u_{0}\right\} \\
\mathcal{F}^{-}\left(u_{0}\right)=\left\{u=w\left(0_{-} ; u_{0}, y\right), y \in \mathbb{R} ; u \neq u_{0}\right\}
\end{array}\right.
$$

that we now characterize.

Lemma 5 We have

$$
\mathcal{F}^{+}\left(u_{0}\right)=\left\{u \neq u_{0} ; \frac{f(v)-f(u)}{v-u}>0 \forall v \in I\left(u, u_{0}\right), v \neq u\right\} .
$$

and

$$
\mathcal{F}^{-}\left(u_{0}\right)=\left\{u \neq u_{0} ; \frac{f(v)-f(u)}{v-u}<0 \forall v \in I\left(u, u_{0}\right), v \neq u\right\} .
$$




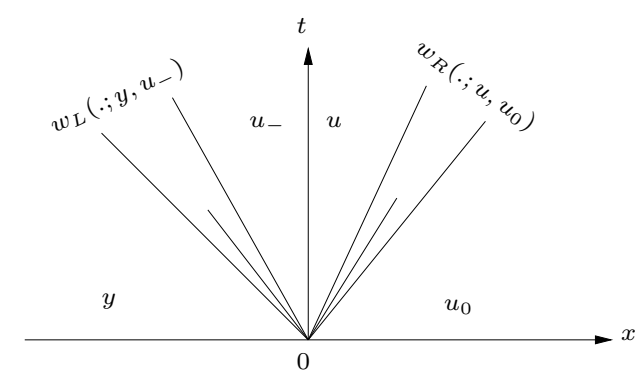

Figure 4: $u \in \mathcal{F}^{+}\left(u_{0}\right)$, i.e. $u=w\left(0_{+} ; y, u_{0}\right), y \in \mathbb{R}$.

Proof. Let us check (23). We first prove

$$
\mathcal{F}^{+}\left(u_{0}\right)=\left\{u \in \mathcal{E}^{+}\left(u_{0}\right) ; w\left(0_{-} ; u, u_{0}\right)=w\left(0_{+} ; u, u_{0}\right)\right\} .
$$

Indeed, let $u \in \mathcal{F}^{+}\left(u_{0}\right)$; clearly $u$ is connected to $u_{0}$ on the left by a wave whose speed is nonnegative (cf. Fig. 4) so that $u \in \mathcal{E}^{+}\left(u_{0}\right)$. In addition, we have

$$
u=w\left(0_{-} ; u, u_{0}\right)=w\left(0_{+} ; u, u_{0}\right) .
$$

Conversely, if $u \in \mathcal{E}^{+}\left(u_{0}\right)$ satisfies (25), $u$ belongs obviously to $\mathcal{F}^{+}\left(u_{0}\right)$. We next show that $u \in \mathcal{E}^{+}\left(u_{0}\right)$ satisfies $(25)$ if and only if

$$
\frac{f(v)-f(u)}{v-u}>0 \quad \forall v \in I\left(u, u_{0}\right), v \neq u .
$$

Observe that the equality $w\left(0_{-} ; u, u_{0}\right)=w\left(0_{+} ; u, u_{0}\right)$ holds if and only if the left subwave of $w\left(\cdot ; u, u_{0}\right)$ is not a stationary shock. Since $u \in \mathcal{E}^{+}\left(u_{0}\right)$, we already know from Lemma 2 that (15) holds and therefore

$$
f^{\prime}(u) \geq 0, \quad \frac{f(u)-f\left(u_{0}\right)}{u-u_{0}} \geq 0 .
$$

Then, if we assume $u>u_{0}$, it is clear geometrically (cf. Fig. 5) that we must have

$$
f(v)<f(u) \quad \forall v \in\left[u_{0}, u\right) .
$$

Indeed, we have a stationary shock if and only if it exists a state $u_{1} \in\left[u_{0}, u\right.$ ) such that $f(u)=f\left(u_{1}\right)$. Hence, there does not exist such a stationary shock if and only if

$$
\frac{f(v)-f(u)}{v-u}>0 \quad \forall v \in\left[u_{0}, u\right) .
$$

Similarly, for $u<u_{0}$, a stationary shock does not exist if and only if

$$
f(v)<f(u) \quad \forall v \in\left(u, u_{0}\right]
$$

or equivalently

$$
\frac{f(v)-f(u)}{v-u}>0 \quad \forall v \in\left(u, u_{0}\right] .
$$

This proves $(23)$. The characterization $(24)$ of $\mathcal{F}^{-}\left(u_{0}\right)$ is obtained analogously. 


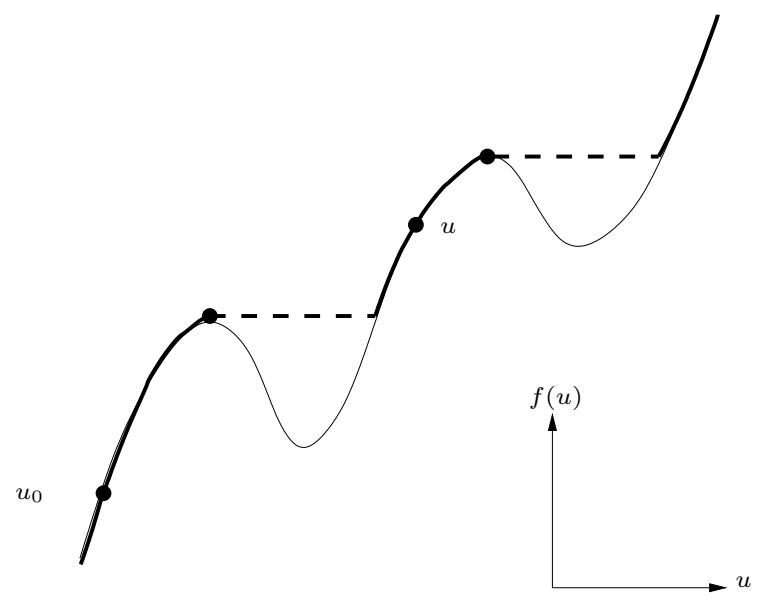

Figure 5: In bold-face, the set $\mathcal{F}^{+}\left(u_{0}\right), u_{0}$ being excluded, and the other circle points being included (note the difference with respect to Fig. 3b). Here $u \in$ $\mathcal{F}^{+}\left(u_{0}\right)$.

Example 2.1. (contd.) If the flux function $f$ is strictly increasing, we have

$$
\mathcal{F}^{+}\left(u_{0}\right)=\mathcal{E}^{+}\left(u_{0}\right)=\mathbb{R} \backslash\left\{u_{0}\right\}, \quad \mathcal{F}^{-}\left(u_{0}\right)=\mathcal{E}^{-}\left(u_{0}\right)=\emptyset
$$

while for a strictly decreasing function $f$

$$
\mathcal{F}^{+}\left(u_{0}\right)=\mathcal{E}^{+}\left(u_{0}\right)=\emptyset, \quad \mathcal{F}^{-}\left(u_{0}\right)=\mathcal{E}^{-}\left(u_{0}\right)=\mathbb{R} \backslash\left\{u_{0}\right\}
$$

Example 2.2. (contd.) Here we can state

Lemma 6 Assume that the function $f$ is strictly convex. Then

$$
\mathcal{F}^{+}\left(u_{0}\right)=\left\{u \neq u_{0} ; u \geq \max \left(\bar{u}, \tilde{u}_{0}\right), u \neq \tilde{u}_{0}\right\}
$$

and

$$
\mathcal{F}^{-}\left(u_{0}\right)=\left\{u \neq u_{0} ; u \leq \min \left(\bar{u}, \tilde{u}_{0}\right), u \neq \tilde{u}_{0}\right\} .
$$

Proof. We check for instance the property (26). It follows from (20) that we have to restrict ourselves to the states $u \geq \max \left(\bar{u}, \tilde{u}_{0}\right)$. Assume first $u \geq \bar{u}$ so that $\bar{u} \geq \tilde{u}_{0}$. Then, we observe that, for $u \geq \bar{u}$, we have indeed

$$
\frac{f(v)-f(u)}{v-u}>0 \quad \forall v \in I\left(u_{0}, u\right), v \neq u
$$

and thus $u \in \mathcal{F}^{-}\left(u_{0}\right)$. Consider next the case $\bar{u} \geq u_{0}$ for which $\tilde{u}_{0} \geq \bar{u}$. For $u \geq \tilde{u}_{0}$, we obtain

$$
\frac{f(v)-f(u)}{v-u}>0 \quad \forall v \in\left[u_{0}, u\right)
$$

if and only if $u>\tilde{u}_{0}$ (cf. Fig. 6) which proves (26).

Now, let $\theta \in C^{1}(\mathbb{R})$ be a strictly monotone function; only for the sake of convenience, we will assume that $\theta$ satisfies $\theta^{\prime}>0$ and maps $\mathbb{R}$ onto itself. We 


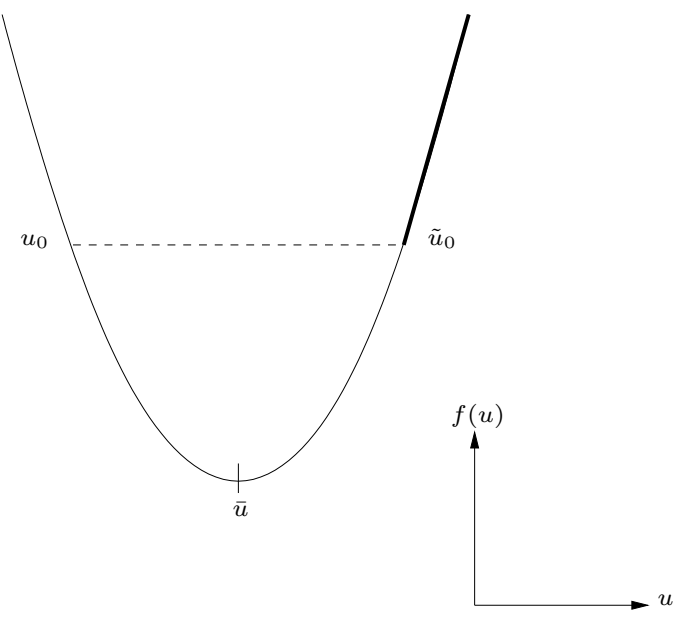

Figure 6: In bold-face, the set $\mathcal{F}^{+}\left(u_{0}\right), \tilde{u}_{0}$ being excluded.

set $\tilde{f}(v)=f(\theta(v))$ and we denote by $z\left(\frac{x}{t} ; v_{g}, v_{d}\right)$ the solution of the Riemann problem expressed in the variable $v=\theta^{-1}(u)$, i.e.,

$$
z\left(\frac{x}{t} ; v_{g}, v_{d}\right)=\theta^{-1}\left(w\left(\frac{x}{t} ; \theta\left(v_{g}\right), \theta\left(v_{d}\right)\right) .\right.
$$

With a given state $v_{0}$ we associate the sets of states

$$
\left\{\begin{array}{l}
\widetilde{\mathcal{E}}^{+}\left(v_{0}\right)=\left\{v=z\left(0_{-} ; y, v_{0}\right), y \in \mathbb{R} ; v \neq v_{0}\right\} \\
\widetilde{\mathcal{F}}^{+}\left(v_{0}\right)=\left\{v=z\left(0_{+} ; y, v_{0}\right), y \in \mathbb{R} ; v \neq v_{0}\right\}
\end{array}\right.
$$

and

$$
\left\{\begin{array}{l}
\widetilde{\mathcal{E}}^{-}\left(v_{0}\right)=\left\{v=z\left(0_{+} ; v_{0}, y\right), y \in \mathbb{R} ; v \neq v_{0}\right\} \\
\widetilde{\mathcal{F}}^{-}\left(v_{0}\right)=\left\{v=z\left(0_{-} ; v_{0}, y\right), y \in \mathbb{R} ; v \neq v_{0}\right\} .
\end{array}\right.
$$

Using Lemmas 2 and 5, we have

$$
\left\{\begin{array}{l}
\widetilde{\mathcal{E}}^{+}\left(v_{0}\right)=\left\{v \neq v_{0} ; \min _{w \in I\left(v_{0}, v\right)} \frac{\tilde{f}(w)-\tilde{f}(v)}{w-v} \geq 0\right\} \\
\widetilde{\mathcal{F}}^{+}\left(v_{0}\right)=\left\{v \neq v_{0} ; \frac{\tilde{f}(w)-\tilde{f}(v)}{w-v}>0 \quad \forall w \in I\left(v_{0}, v\right), w \neq v\right\}
\end{array}\right.
$$

and

$$
\left\{\begin{array}{l}
\widetilde{\mathcal{E}}^{-}\left(v_{0}\right)=\left\{v \neq v_{0} ; \max _{w \in I\left(v_{0}, v\right)} \frac{\tilde{f}(w)-\tilde{f}(v)}{w-v} \leq 0\right\} \\
\widetilde{\mathcal{F}}^{-}\left(v_{0}\right)=\left\{v \neq v_{0} ; \frac{\tilde{f}(w)-\tilde{f}(v)}{w-v}<0 \quad \forall w \in I\left(v_{0}, v\right), w \neq v\right\} .
\end{array}\right.
$$


Example 2.1. (contd.) If the function $f$ is strictly increasing, we have

$$
\widetilde{\mathcal{E}}^{+}\left(v_{0}\right)=\widetilde{\mathcal{F}}^{+}\left(v_{0}\right)=\mathbb{R} \backslash\left\{v_{0}\right\}, \quad \widetilde{\mathcal{E}}^{-}\left(v_{0}\right)=\widetilde{\mathcal{F}}^{-}\left(v_{0}\right)=\emptyset
$$

while if $f$ is strictly decreasing

$$
\widetilde{\mathcal{E}}^{+}\left(v_{0}\right)=\widetilde{\mathcal{F}}^{+}\left(v_{0}\right)=\emptyset, \quad \widetilde{\mathcal{E}}^{-}\left(v_{0}\right)=\widetilde{\mathcal{F}}^{-}\left(v_{0}\right)=\mathbb{R} \backslash\left\{v_{0}\right\} .
$$

Example 2.2. (contd.) When the function $f$ is strictly convex, we denote by $\bar{v}=\theta^{-1}(\bar{u})$ the sonic state of $\tilde{f}$. Given a state $v_{0}$, we set $u_{0}=\theta\left(v_{0}\right)$ and $\tilde{v}_{0}=\theta^{-1}\left(\tilde{u}_{0}\right)$. Then we obtain

$$
\widetilde{\mathcal{E}}^{+}\left(v_{0}\right)=\left\{v \neq v_{0} ; v \geq \max \left(\bar{v}, \tilde{v}_{0}\right)\right\}, \quad \widetilde{\mathcal{F}}^{+}\left(v_{0}\right)=\left\{v \in \widetilde{\mathcal{E}}^{+}\left(v_{0}\right) ; v \neq \tilde{v}_{0}\right\}
$$

and

$$
\widetilde{\mathcal{E}}^{-}\left(v_{0}\right)=\left\{v \neq v_{0} ; v \leq \min \left(\bar{v}, \tilde{v}_{0}\right)\right\}, \quad \widetilde{\mathcal{F}}^{-}\left(v_{0}\right)=\left\{v \in \widetilde{\mathcal{E}}^{-}\left(v_{0}\right) ; v \neq \tilde{v}_{0}\right\} .
$$

\section{$2.2 \quad \mathrm{v}$-continuous solutions.}

Let us now look for all possible self-similar solutions $u=u\left(\frac{x}{t}\right)$ of the coupled Riemann problem. Again, for convenience, we assume that both functions $\theta_{L}$ and $\theta_{R}$ are strictly increasing and map $\mathbb{R}$ onto itself. We begin with those selfsimilar solutions which are $v$-continuous at the interface (in the strong sense), i.e., which satisfy

$$
v\left(0_{-}\right)=v\left(0_{+}\right)=v(0)
$$

where $v$ is defined from $u$ as in (6), or equivalently which satisfy the constraint

$$
v(0) \in\left(\left\{v_{g}\right\} \cup \widetilde{\mathcal{F}}_{L}^{-}\left(v_{g}\right)\right) \cap\left(\left\{v_{d}\right\} \cup \widetilde{\mathcal{F}}_{R}^{+}\left(v_{d}\right)\right) .
$$

Hence, besides the trivial solution corresponding to $v(0)=v_{g}=v_{d}{ }^{2}$, we obtain three types of v-continuous solutions.

(i) The first type of v-continuous solution. If

$$
v(0)=v_{d} \in \widetilde{\mathcal{F}}_{L}^{-}\left(v_{g}\right),
$$

the solution of the coupled Riemann problem coincides with the solution $z_{L}\left(\cdot ; v_{g}, v_{d}\right)$ of the L-Riemann problem: it consists of a (composite) L-wave whose speed is nonpositive. Such a solution is characterized by

$$
\frac{\tilde{f}_{L}(v)-\tilde{f}_{L}\left(v_{d}\right)}{v-v_{d}}<0 \quad \forall v \in I\left(v_{g}, v_{d}\right), v \neq v_{d} .
$$

(ii) The second type of v-continuous solution. If

$$
v(0)=v_{g} \in \widetilde{\mathcal{F}}_{R}^{+}\left(v_{d}\right),
$$

\footnotetext{
${ }^{2}$ which is excluded since, once for all, we have supposed $v_{g} \neq v_{d}$.
} 
the solution of the coupled Riemann problem coincides with the solution $z_{R}\left(\cdot ; v_{g}, v_{d}\right)$ of the R-Riemann problem: it consists of a (composite) R-wave whose speed is nonnegative. Such a solution is characterized by

$$
\frac{\tilde{f}_{R}(v)-\tilde{f}_{R}\left(v_{g}\right)}{v-v_{g}}>0 \quad \forall v \in I\left(v_{g}, v_{d}\right), v \neq v_{g} .
$$

(iii) The third type of v-continuous solution. The general case is indeed obtained by choosing

$$
v(0) \in \widetilde{\mathcal{F}}_{L}^{-}\left(v_{g}\right) \cap \widetilde{\mathcal{F}}_{R}^{+}\left(v_{d}\right)
$$

Obviously, this requires the condition

$$
\widetilde{\mathcal{F}}_{L}^{-}\left(v_{g}\right) \cap \widetilde{\mathcal{F}}_{R}^{+}\left(v_{d}\right) \neq \emptyset .
$$

Then a solution of the coupled Riemann problem coincides with $z_{L}\left(\cdot ; v_{g}, v(0)\right)$ in the domain $(x<0, t>0)$ and with $z_{R}\left(\cdot ; v(0), v_{d}\right)$ in the domain $(x>, t>0)$. It consists of two (composite) waves: a L-wave whose speed is nonpositive and a $\mathrm{R}$-wave whose speed is nonnegative. Such a solution is characterized by the conditions

$$
\begin{cases}\frac{\tilde{f}_{L}(v)-\tilde{f}_{L}(v(0))}{v-v(0)}<0 & \forall v \in I\left(v_{g}, v(0)\right), v \neq v(0) \\ \frac{\tilde{f}_{R}(v)-\tilde{f}_{R}(v(0))}{v-v(0)}>0 & \forall v \in I\left(v(0), v_{d}\right), v \neq v(0) .\end{cases}
$$

We thus find a one-parameter family of solutions depending on the parameter $v(0) \in \widetilde{\mathcal{F}}_{L}^{-}\left(v_{g}\right) \cap \widetilde{\mathcal{F}}_{R}^{-}\left(v_{d}\right)$.

Let us notice that a solution of type (i) or type (ii) is that of a classical L or R-Riemann problem and may be viewed as a quasi trivial solution of this coupling problem.

We now apply these results to the case where both flux functions $f_{L}$ and $f_{R}$ are either strictly monotone or strictly convex.

Example 2.3. The case of strictly monotone flux functions.

(a) Suppose first that the functions $f_{L}$ and $f_{R}$ are strictly decreasing so that

$$
\widetilde{\mathcal{F}}_{L}^{-}\left(v_{g}\right)=\mathbb{R} \backslash\left\{v_{g}\right\}, \quad \widetilde{\mathcal{F}}_{R}^{+}\left(v_{d}\right)=\emptyset .
$$

Then, clearly the solution of type (i) alone is admissible.

(b) Similarly, if the functions $f_{L}$ and $f_{R}$ are strictly increasing so that

$$
\widetilde{\mathcal{F}}_{L}^{-}\left(v_{g}\right)=\emptyset, \quad \widetilde{\mathcal{F}}_{R}^{+}\left(v_{d}\right)=\mathbb{R} \backslash\left\{v_{d}\right\},
$$

the solution of type (ii) alone is admissible.

(c) Suppose next that $f_{L}$ is strictly increasing and $f_{R}$ is strictly decreasing. We have $\widetilde{\mathcal{F}}_{L}^{-}\left(v_{g}\right)=\widetilde{\mathcal{F}}_{R}^{+}\left(v_{d}\right)=\emptyset$. Then, none of the existence conditions of a v-continuous solution holds: there does not exist any v-continuous solution of the coupled Riemann problem (except the trivial solution corresponding to 
$\left.v(0)=v_{g}=v_{d}\right)$.

(d) If $f_{L}$ is strictly decreasing and $f_{R}$ is strictly increasing, we have

$$
\tilde{\mathcal{F}}_{L}^{-}\left(v_{g}\right)=\mathbb{R} \backslash\left\{v_{g}\right\}, \quad \widetilde{\mathcal{F}}_{R}^{+}\left(v_{d}\right)=\mathbb{R} \backslash\left\{v_{d}\right\} .
$$

Hence any above condition of existence of a v-continuous solution holds: there exists a one-parameter family of solutions of type (iii) depending on the parameter $v(0) \in \mathbb{R}$. Clearly this family contains the solution of type (i) and that of type (ii). Hence the coupled Riemann problem has an infinite number of $\mathrm{v}$ continuous solutions and it is enough to specify $v(0)$ for determining the unique corresponding solution.

Example 2.4. The case of strictly convex flux functions with sonic states.

Here we assume that $f_{\alpha}, \alpha=L, R$, is a strictly convex function and possesses a sonic state $\bar{u}_{\alpha}$. We set: $\bar{v}_{\alpha}=\theta_{\alpha}^{-1}\left(\bar{u}_{\alpha}\right)$. With the pair $\left(v_{g}, v_{d}\right)$, we associate the pair $\left(\tilde{v}_{g}, \tilde{v}_{d}\right)$ defined by

$$
\left\{\begin{array}{l}
\tilde{f}_{L}\left(\tilde{v}_{g}\right)=\tilde{f}_{L}\left(v_{g}\right), \tilde{v}_{g} \neq v_{g} \text { if } v_{g} \neq \bar{v}_{L} \\
\tilde{v}_{g}=\bar{v}_{L} \text { if } v_{g}=\bar{v}_{L}
\end{array},\left\{\begin{array}{l}
\tilde{f}_{R}\left(\tilde{v}_{d}\right)=\tilde{f}_{R}\left(v_{d}\right), \tilde{v}_{d} \neq v_{d} \text { if } v_{d} \neq \bar{v}_{R} \\
\tilde{v}_{d}=\bar{v}_{R} \text { if } v_{d}=\bar{v}_{R} .
\end{array}\right.\right.
$$

Using the results of Example 2.2, we thus have

$$
\begin{aligned}
\widetilde{\mathcal{F}}_{L}^{-}\left(v_{g}\right) & =\left\{v \neq v_{g} ; v<\min \left(\bar{v}_{L}, \tilde{v}_{g}\right), v \neq \tilde{v}_{g}\right\} \\
\widetilde{\mathcal{F}}_{R}^{+}\left(v_{d}\right) & =\left\{v \neq v_{d} ; v>\max \left(\bar{v}_{R}, \tilde{v}_{d}\right), v \neq \tilde{v}_{d}\right\} .
\end{aligned}
$$

(a) If

$$
v_{d} \in \widetilde{\mathcal{F}}_{L}^{-}\left(v_{g}\right) \Leftrightarrow v_{d} \leq \min \left(\bar{v}_{L}, \tilde{v}_{g}\right), v_{d} \neq \tilde{v}_{g},
$$

there exists a solution of type (i) (a L-wave) to the coupled Riemann problem. This is the only solution of this kind.

(b) If

$$
v_{g} \in \widetilde{\mathcal{F}}_{R}^{+}\left(v_{g}\right) \Leftrightarrow v_{g} \geq \max \left(\bar{v}_{R}, \tilde{v}_{d}\right), v_{g} \neq \tilde{v}_{d},
$$

there exists a solution of type (ii) (a R-wave) to the coupled Riemann problem. This is the only solution of this type.

(c) When

$$
\widetilde{\mathcal{F}}_{L}^{-}\left(v_{g}\right) \cap \widetilde{\mathcal{F}}_{R}^{+}\left(v_{d}\right) \neq \emptyset \Leftrightarrow \max \left(\bar{v}_{R}, \tilde{v}_{d}\right) \leq \min \left(\bar{v}_{L}, \tilde{v}_{g}\right),
$$

we can construct a family of solutions of type (iii) (a L-wave followed by a Rwave) depending on the parameter $v(0) \in\left[\max \left(\bar{v}_{R}, \tilde{v}_{d}\right), \min \left(\bar{v}_{L}, \tilde{v}_{g}\right)\right]$. They are the only solutions of type (iii).

It is worthwile to notice that, given a pair $\left(v_{g}, v_{d}\right)$, we may have v-continuous solutions of several types. For instance, if

$$
v_{d} \leq \max \left(\bar{v}_{R}, \tilde{v}_{d}\right) \leq \min \left(\bar{v}_{L}, \tilde{v}_{g}\right)
$$

solutions of types (i) and (iii) are valid. 


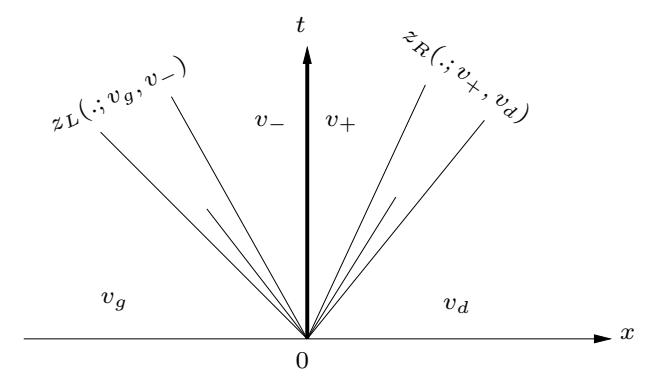

Figure 7: A v-discontinuous solution to the coupled Riemann problem

\section{$2.3 \quad$ v-discontinuous solutions.}

We next look for the self-similar solutions of the coupled Riemann problem which are $v$-discontinuous at the interface $x=0$. Setting

$$
v_{-}=v\left(0_{-}\right), \quad v_{+}=v\left(0_{+}\right),
$$

the coupling constraints (7) read here

$$
\left\{\begin{array}{c}
v_{-} \in \widetilde{\mathcal{O}}_{L}\left(v_{+}\right) \Leftrightarrow v_{-}=z_{L}\left(0_{-} ; v_{-}, v_{+}\right), \\
v_{+} \in \widetilde{\mathcal{O}}_{R}\left(v_{-}\right) \Leftrightarrow v_{+}=z_{R}\left(0_{+} ; v_{-}, v_{+}\right) .
\end{array}\right.
$$

Since we assume $v_{-} \neq v_{+}, z_{L}\left(\cdot ; v_{-}, v_{+}\right)$and $z_{R}\left(\cdot ; v_{-}, v_{+}\right)$are both non trivial waves. The coupling constraints mean that $z_{L}\left(\cdot ; v_{-}, v_{+}\right)$is a wave with a nonnegative speed while $z_{R}\left(\cdot ; v_{-}, v_{+}\right)$is a wave with a nonpositive speed.

On the other hand, any solution of the coupled Riemann problem consists necessarily of a L-wave whose speed is nonpositive and a $\mathrm{R}$-wave whose speed is nonnegative. In other words, the wave $z_{L}\left(\cdot ; v_{g}, v_{-}\right)$has a nonpositive speed while $z_{R}\left(\cdot ; v_{+}, v_{d}\right)$ has a non negative speed (see Fig. 7 ).

Let us then state

Lemma 7 One of the two following situations holds:

(i) $v_{-}=v_{g} \Rightarrow \tilde{f}_{L}^{\prime}\left(v_{g}\right) \geq 0$;

(ii) $v_{-} \neq v_{g} \Rightarrow \tilde{f}_{L}^{\prime}\left(v_{-}\right)=0$ and the right subwave of $z_{L}\left(\cdot ; v_{g}, v_{-}\right)$is a rarefaction.

In the case (ii), it is worthwile to notice that $v_{-}$is a sonic state of the right rarefaction subwave of $z_{L}\left(\cdot ; v_{g}, v_{-}\right)$.

Proof. We begin by proving the lemma when $\theta_{L}=i d$, i.e., when $v=u$ is the conservative variable. Since $w_{L}\left(\cdot ; u_{-}, u_{+}\right)$has a nonnegative speed, we have

$$
\min _{u \in I\left(u_{-}, u_{+}\right)} \frac{f_{L}(u)-f_{L}\left(u_{-}\right)}{u-u_{-}} \geq 0
$$

which implies $f_{L}^{\prime}\left(u_{-}\right) \geq 0$. If we assume $u_{-}=u_{g}$, we obtain $f_{L}^{\prime}\left(u_{g}\right) \geq 0$. Assume next $u_{-} \neq u_{g}$. Then $w_{L}\left(\cdot ; u_{g}, u_{-}\right)$has a nonpositive speed so that

$$
\max _{u \in I\left(u_{g}, u_{-}\right)} \frac{f_{L}(u)-f_{L}\left(u_{-}\right)}{u-u_{-}} \leq 0
$$




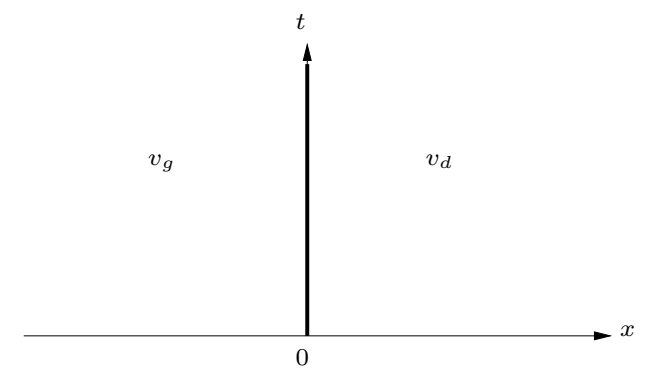

Figure 8: The first type of v-discontinuous solution : a stationary discontinuity.

which yields $f_{L}^{\prime}\left(u_{-}\right) \leq 0$. Hence we find $f_{L}^{\prime}\left(u_{-}\right)=0$. As a consequence, the right subwave of $w_{L}\left(\cdot ; u_{g}, u_{-}\right)$is either a rarefaction with $u_{-}$as a sonic state or a stationary shock. But, since $w_{L}\left(0_{-} ; u_{g}, u_{-}\right)=u_{-}$, a stationary shock is not allowed. This proves the lemma when $v=u$.

Let us now turn to the general case of a nonconservative variable $v$. Since $\tilde{f}_{L}^{\prime}(v)=f_{L}^{\prime}\left(\theta_{L}(v)\right) \theta_{L}^{\prime}(v)$ and $\theta_{L}^{\prime}(v)>0$, the above properties (i) and (ii) become respectively

$$
\begin{aligned}
& v_{-}=v_{g} \Rightarrow \tilde{f}_{L}^{\prime}\left(v_{g}\right) \geq 0 \\
& v_{-} \neq v_{g} \Rightarrow \tilde{f}_{L}^{\prime}\left(v_{-}\right)=0
\end{aligned}
$$

and the proof is complete.

Similarly, one can state

Lemma 8 One of the two following situations holds:

(i) $v_{+}=v_{d} \Rightarrow \tilde{f}_{R}^{\prime}\left(v_{d}\right) \leq 0$;

(ii) $v_{+} \neq v_{d} \Rightarrow \tilde{f}_{R}^{\prime}\left(v_{+}\right)=0$ and the left subwave of $z_{R}\left(\cdot ; v_{+}, v_{d}\right)$ is a rarefaction.

As a consequence of Lemmas 7 and 8, we find that the self-similar v-discontinuous solutions of the coupled Riemann problem are necessarily of the four following types.

(i) The first type of v-discontinuous solution (see Fig. 8). It consists of a stationary discontinuity with $v_{-}=v_{g}$ and $v_{+}=v_{d}$. Such a solution exists if and only if we have

$$
\tilde{f}_{L}^{\prime}\left(v_{g}\right) \geq 0 \geq \tilde{f}_{R}^{\prime}\left(v_{d}\right)
$$

together with the coupling conditions which read here

$$
\begin{aligned}
& \min _{v \in I\left(v_{g}, v_{d}\right)} \frac{\tilde{f}_{L}(v)-\tilde{f}_{L}\left(v_{g}\right)}{v-v_{g}} \geq 0, \\
& \max _{v \in I\left(v_{g}, v_{d}\right)} \frac{\tilde{f}_{R}(v)-\tilde{f}_{R}\left(v_{d}\right)}{v-v_{d}} \leq 0 .
\end{aligned}
$$

(ii) The second type of v-discontinuous solution (see Fig. 9). It consists of a L-wave whose right subwave is a rarefaction with $v_{-}$as a sonic state followed 


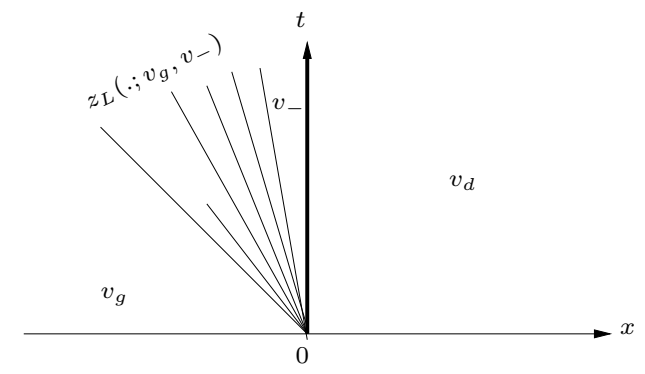

Figure 9: The second type of v-discontinuous solution : a $L$-wave whose right subwave is a rarefaction, followed by a stationary discontinuity.

by a stationary discontinuity with $v_{+}=v_{d}$. Such a solution exists under the following conditions. On the one hand, we have

$$
\tilde{f}_{R}^{\prime}\left(v_{d}\right) \leq 0
$$

and there exists a sonic state $\bar{v}_{L}$ of $\tilde{f}_{L}, \bar{v}_{L} \neq v_{g}$, such that

$$
\max _{v \in I\left(v_{g}, \bar{v}_{L}\right)} \frac{\tilde{f}_{L}(v)-\tilde{f}_{L}\left(\bar{v}_{L}\right)}{v-\bar{v}_{L}}=\tilde{f}_{L}^{\prime}\left(\bar{v}_{L}\right)=0
$$

and $v_{-}=\bar{v}_{L}$. On the other hand, we require the associated coupling conditions

$$
\begin{aligned}
& \min _{v \in I\left(\bar{v}_{L}, v_{d}\right)} \frac{\tilde{f}_{L}(v)-\tilde{f}_{L}\left(\bar{v}_{L}\right)}{v-\bar{v}_{L}} \geq 0, \\
& \max _{v \in I\left(\bar{v}_{L}, v_{d}\right)} \frac{\tilde{f}_{R}(v)-\tilde{f}_{R}\left(v_{d}\right)}{v-v_{d}} \leq 0 .
\end{aligned}
$$

(iii) The third type of v-discontinuous solution (see Fig. 10). It consists of a stationary discontinuity with $v_{-}=v_{g}$ followed by a R-wave whose left subwave is a rarefaction with $v_{+}$as a sonic state. This solution exists under the following conditions. On the one hand, we have

$$
\tilde{f}_{L}^{\prime}\left(v_{g}\right) \geq 0
$$

and there exists a sonic state $\bar{v}_{R}$ of $\tilde{f}_{R}, \bar{v}_{R} \neq v_{d}$ such that

$$
\min _{v \in I\left(\bar{v}_{R}, v_{d}\right)} \frac{\tilde{f}_{R}(v)-\tilde{f}_{R}\left(\bar{v}_{R}\right)}{v-\bar{v}_{R}}=\tilde{f}_{R}^{\prime}\left(\bar{v}_{R}\right)=0
$$

and $v_{+}=\bar{v}_{R}$. On the other hand, the associated coupling conditions read

$$
\begin{gathered}
\min _{v \in I\left(v_{g}, \bar{v}_{R}\right)} \frac{\tilde{f}_{L}(v)-\tilde{f}_{L}\left(v_{g}\right)}{v-v_{g}} \geq 0, \\
\max _{v \in I\left(v_{g}, \bar{v}_{R}\right)} \frac{\tilde{f}_{R}(v)-\tilde{f}_{R}\left(\bar{v}_{R}\right)}{v-\bar{v}_{R}} \leq 0 .
\end{gathered}
$$




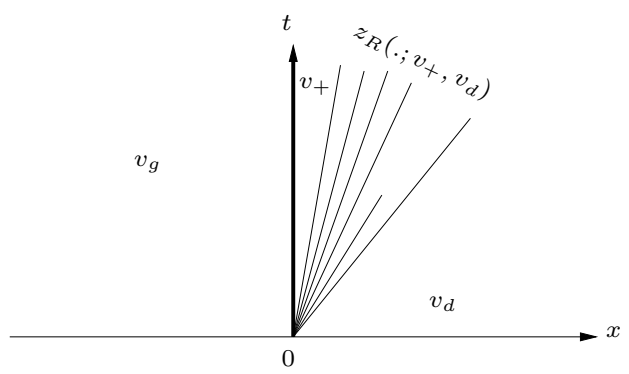

Figure 10: The third type of v-discontinuous solution : a stationary discontinuity followed by a $R$-wave whose left subwave is a rarefaction.

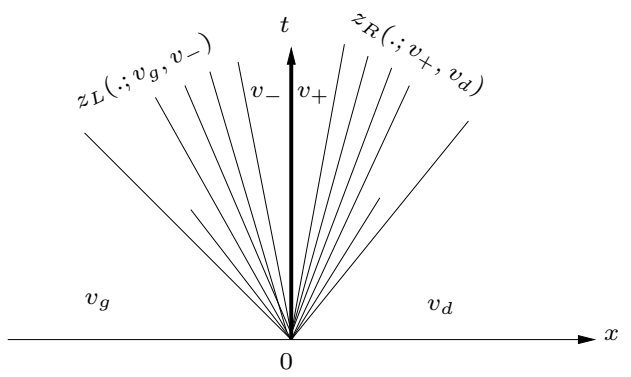

Figure 11: The fourth type of v-discontinuous solution : a $L$-wave whose right subwave is a rarefaction, followed by a stationary discontinuity, itself followed by a $R$-wave whose left subwave is a rarefaction.

(iv) The fourth type of v-discontinuous solution (see Fig. 11). It consists of a L-wave whose right subwave is a rarefaction with $v_{-}$as a sonic state followed by a stationary discontinuity and a R-wave whose left subwave is a rarefaction with $v_{+}$as a sonic state. For obtaining such a solution, the following conditions hold: there exist sonic states $\bar{v}_{L} \neq v_{g}$ and $\left.\bar{v}_{R}\right) \neq v_{d}$ of $\tilde{f}_{L}$ and $\tilde{f}_{R}$ respectively such that

$$
\begin{aligned}
& \max _{v \in I\left(v_{g}, \bar{v}_{L}\right)} \frac{\tilde{f}_{L}(v)-\tilde{f}_{L}\left(\bar{v}_{L}\right)}{v-\bar{v}_{L}}=\tilde{f}_{L}^{\prime}\left(\bar{v}_{L}\right)=0, \\
& \min _{v \in I\left(\bar{v}_{R}, v_{d}\right)} \frac{\tilde{f}_{R}(v)-\tilde{f}_{R}\left(\bar{v}_{R}\right)}{v-\bar{v}_{R}}=\tilde{f}_{R}^{\prime}\left(\bar{v}_{R}\right)=0
\end{aligned}
$$

and $v_{-}=\bar{v}_{L}, v_{+}=\bar{v}_{R}$. In addition, we require the coupling conditions

$$
\min _{v \in I\left(\bar{v}_{L}, \bar{v}_{R}\right)} \frac{\tilde{f}_{L}(v)-\tilde{f}_{L}\left(\bar{v}_{L}\right)}{v-\bar{v}_{L}} \geq 0
$$

and

$$
\max _{v \in I\left(\bar{v}_{L}, \bar{v}_{R}\right)} \frac{\tilde{f}_{R}(v)-\tilde{f}_{R}\left(\bar{v}_{R}\right)}{v-\bar{v}_{R}} \leq 0 .
$$

Again we apply the above results to the cases where both flux functions are 
either strictly monotone or strictly convex.

Example 2.3. The case of strictly monotone flux functions. (contd.)

(a) If the functions $f_{L}$ and $f_{R}$ are strictly decreasing, we have $\tilde{f}_{L}^{\prime} \leq 0$ and $\tilde{f}_{R}^{\prime} \leq 0$ and no v-discontinuous solution can exist. This is obvious for solutions of types (i) and (iii). On the other hand, due to the coupling conditions, solutions of types (ii) and (iv) are not admissible. For instance, in the case of a solution of type (ii), the first coupling condition implies the existence of a sonic state $\bar{v}_{L}$ such that $\bar{v}_{L}=v_{d}$, i.e., $v_{-}=v_{+}$which is clearly excluded.

(b) If $f_{L}$ and $f_{R}$ are strictly increasing, a similar analysis shows again that there cannot exist any v- discontinuous solution.

(c) If $f_{L}$ is strictly increasing and $f_{R}$ is strictly decreasing, only the v-discontinuous solution of type (i), i.e., a stationary discontinuity, is admissible. Indeed, such a solution is clearly admissible. On the other hand, a solution of type (ii) cannot exist since the condition

$$
\max _{v \in I\left(v_{g}, \bar{v}_{L}\right)} \frac{\tilde{f}_{L}(v)-\tilde{f}_{L}\left(\bar{v}_{L}\right)}{v-\bar{v}_{L}}=\tilde{f}_{L}^{\prime}\left(\bar{v}_{L}\right)=0
$$

implies $\bar{v}_{L}=v_{g}$ and therefore $v_{-}=v_{g}$ so that the L-wave does not exist. Using similar arguments, one can check that the solutions of types (iii) and (iv) are also excluded.

(d) If $f_{L}$ is strictly decreasing and $f_{R}$ is strictly increasing, no v-discontinuous solution may exist since the coupling conditions are never satisfied.

To summarize, a v-discontinuous solution exists only when $f_{L}$ is strictly increasing and $f_{R}$ is strictly decreasing . This is a stationary discontinuity.

Example 2.4.(contd.) Consider again the case where both flux functions $f_{L}$ and $f_{R}$ are strictly convex and possess sonic states $\bar{u}_{L}$ and $\bar{u}_{R}$ respectively. Since, for $\alpha=L, R$, the function $\theta_{\alpha}$ is assumed to satisfy $\theta_{\alpha}^{\prime}>0$, the function $\tilde{f}_{\alpha}$ has a unique sonic state $\bar{v}_{\alpha}=\theta_{\alpha}^{-1}\left(\bar{u}_{\alpha}\right)$ and is strictly decreasing in $\left(-\infty, \bar{v}_{\alpha}\right)$ (resp. strictly increasing in $\left.\left(\bar{v}_{\alpha},+\infty\right)\right)$. We introduce again the states $\tilde{v}_{g}$ and $\tilde{v}_{d}$ defined above. Note that, in this strictly convex case, the sonic state $\bar{v}_{L} \neq v_{g}$ of $\tilde{f}_{L}$ satisfies the condition

$$
\max _{v \in I\left(v_{g}, \bar{v}_{L}\right)} \frac{\tilde{f}_{L}(v)-\tilde{f}_{L}\left(v_{g}\right)}{v-v_{g}}=\tilde{f}_{L}^{\prime}\left(\bar{v}_{L}\right)=0
$$

if and only if $v_{g}<\bar{v}_{L}$ or equivalently $\tilde{f}_{L}^{\prime}\left(v_{g}\right)<0$. Similarly, the sonic state $\bar{v}_{R} \neq v_{d}$ of $\tilde{f}_{R}$ satisfies the condition

$$
\min _{v \in I\left(\bar{v}_{R}, v_{d}\right)} \frac{\tilde{f}_{R}(v)-\tilde{f}_{R}\left(v_{d}\right)}{v-v_{d}}=\tilde{f}_{R}^{\prime}\left(\bar{v}_{R}\right)=0
$$

if and only if $v_{d}>\bar{v}_{R}$ or equivalently $\tilde{f}_{R}^{\prime}\left(v_{d}\right)>0$.

On the other hand, for $v_{a}=v_{g} \geq \bar{v}_{L}$ or $v_{a}=\bar{v}_{L}$, a coupling condition of the form

$$
\min _{I\left(v_{a}, v_{b}\right)} \frac{\tilde{f}_{L}(v)-\tilde{f}_{L}\left(v_{a}\right)}{v-v_{a}} \geq 0
$$


holds if and only if $v_{b} \geq \tilde{v}_{a}$ where

$$
\tilde{v}_{a}= \begin{cases}\tilde{v}_{g} & \text { if } v_{a}=v_{g} \\ \bar{v}_{L} & \text { if } v_{a}=\bar{v}_{L} .\end{cases}
$$

Similarly, for $v_{b}=v_{d} \leq \bar{v}_{R}$ or $v_{b}=\bar{v}_{R}$, a coupling condition of the form

$$
\max _{I\left(v_{a}, v_{b}\right)} \frac{\tilde{f}_{R}(v)-\tilde{f}_{R}\left(v_{b}\right)}{v-v_{b}} \leq 0
$$

holds if and only if $v_{a} \leq \tilde{v}_{b}$ where

$$
\tilde{v}_{b}= \begin{cases}\tilde{v}_{d} & \text { if } v_{b}=v_{d} \\ \bar{v}_{R} & \text { if } v_{b}=\bar{v}_{R} .\end{cases}
$$

Then, it is an easy matter to check that a v-discontinuous solution exists in the following situations.

(a) If

$$
\bar{v}_{L} \leq v_{g} \leq \tilde{v}_{d}, \quad \tilde{v}_{g} \leq v_{d} \leq \bar{v}_{R}
$$

we obtain a v-discontinuous solution of type (i), i.e., a stationary discontinuity. (b) If

$$
v_{g}<\bar{v}_{L}<v_{d} \leq \bar{v}_{R},
$$

we find a v-discontinuous solution of type (ii), i.e., a L-wave followed by a stationary discontinuity.

(c) If

$$
\bar{v}_{L} \leq v_{g}<\bar{v}_{R}<v_{d},
$$

we obtain a v-discontinuous solution of type (iii), i.e., a stationary discontinuity followed by a R-wave.

(d) If

$$
v_{g}<\bar{v}_{L}<\bar{v}_{R}<v_{d},
$$

we find a v-discontinuous solution of type (iv), i.e., a L-wave followed by a stationary discontinuity and a $\mathrm{R}$-wave.

Note that each case (i)-(iv) is disclosed from the others and each v-discontinuous solution is uniquely defined.

\subsection{Solution of the coupled Riemann problem.}

We are now able to solve the coupled Riemann problem for all pair $\left(u_{g}, u_{d}\right)$ or $\left(v_{g}, v_{d}\right)$. We begin with the cases where the flux functions $f_{\alpha}, \alpha=L, R$, are either strictly monotone or strictly convex.

Example 2.3.(contd.) We first assume that $f_{\alpha}, \alpha=L, R$ is a strictly monotone function. Combining the above results, we obtain the following conclusions.

(a) The functions $f_{L}$ and $f_{R}$ are strictly decreasing. The solution is v-continuous: it is a L-wave.

(b) The functions $f_{L}$ and $f_{R}$ are strictly increasing. The solution is v-continuous: it is a $\mathrm{R}$-wave.

(c) The function $f_{L}$ is strictly increasing and the function $f_{R}$ strictly decreasing. The solution is v- discontinuous: it is a stationary discontinuity. 
(d) The function $f_{L}$ is strictly decreasing and the function $f_{R}$ is strictly increasing. The solutions are v- continuous and form a one-parameter family depending on thre parameter $v(0) \in \mathbb{R}$. For $v(0) \neq v_{g}, v_{d}$, we obtain a L-wave followed by a R-wave. For $v(0)=v_{d}$, we obtain a L-wave while, for $v(0)=v_{g}$, we get a R-wave.

To summarize, in this case, the coupled Riemann problem has always a solution. This solution is unique except in the subcase (d). Note that, as in [19], one could have obtained directly the above results by using a method of characteristics.

Example 2.4.(contd.) Assume now that the flux functions $f_{L}$ and $f_{R}$ are strictly convex and possess sonic states $\bar{u}_{L}$ and $\bar{u}_{R}$ respectively.Let us check that the coupled Riemann problem has at least one solution. First of all, we already know from the results of section 2.1.2 that a v-continuous solution exists in the following cases.

(a) For $v_{d} \leq \min \left(\bar{v}_{L}, \tilde{v}_{g}\right), \quad v_{d} \neq \tilde{v}_{g}$, the solution is a L-wave.

(b) For $v_{g} \geq \max \left(\bar{v}_{R}, \tilde{v}_{d}\right), \quad v_{g} \neq \tilde{v}_{d}$, the solution is a R-wave.

(c) If $\max \left(\bar{v}_{R}, \tilde{v}_{d}\right) \leq \min \left(\bar{v}_{L}, \tilde{v}_{g}\right)$, we obtain a family of $\mathrm{v}$-continuous solutions consisting of a L-wave followed by a R-wave and depending on the parameter $v(0) \in\left[\max \left(\bar{v}_{R}, \tilde{v}_{d}\right), \min \left(\bar{v}_{L}, \tilde{v}_{g}\right)\right]$.

It remains to exhibit a v-discontinuous solution when a $\mathrm{v}$ - continuous one does not exist, i.e., when the pair $\left(v_{g}, v_{d}\right)$ satisfies the conditions

$$
\left\{\begin{array}{l}
v_{d}>\min \left(\bar{v}_{L}, \tilde{v}_{g}\right) \\
v_{g}<\max \left(\bar{v}_{R}, \tilde{v}_{d}\right) \\
\max \left(\bar{v}_{R}, \tilde{v}_{d}\right)>\min \left(\bar{v}_{L}, \tilde{v}_{g}\right) .
\end{array}\right.
$$

In fact, it is convenient to distinguish the following cases:

$\left(v_{g} \geq \bar{v}_{L}, v_{d} \leq \bar{v}_{R}\right),\left(v_{g} \geq \bar{v}_{L}, v_{d}>\bar{v}_{R}\right),\left(v_{g}<\bar{v}_{L}, v_{d} \leq \bar{v}_{R}\right),\left(v_{g}<\bar{v}_{L}, v_{d}>\bar{v}_{R}\right)$.

(d) For $\left(v_{g} \geq \bar{v}_{L}, v_{d} \leq \bar{v}_{R}\right)$, the conditions (34) become respectively

$$
v_{d}<\tilde{v}_{g}, \quad v_{g}<\tilde{v}_{d}, \quad \tilde{v}_{d}>\tilde{v}_{g} .
$$

This case is therefore characterized by

$$
\bar{v}_{L} \leq v_{g}<\tilde{v}_{d}, \quad \tilde{v}_{g}<v_{d} \leq \bar{v}_{R}
$$

Then, applying the results of section 2.1.3, we obtain that the solution of the coupled Riemann problem is a stationary discontinuity.

(e) For $\left(v_{g} \geq \bar{v}_{L}, v_{d}>\bar{v}_{R}\right)$, the conditions (34) read

$$
v_{d}>\tilde{v}_{g}, \quad v_{g}<\bar{v}_{R}, \quad \bar{v}_{R}>\tilde{v}_{g}
$$

so that this case is characterized by

$$
\bar{v}_{L} \leq v_{g}<\bar{v}_{R}<v_{d}
$$

This implies that the solution is a L-wave followed by a stationary discontinuity.

(f) For $\left(v_{g}<\bar{v}_{L}, v_{d} \leq \bar{v}_{R}\right)$, (34) gives

$$
v_{d}>\bar{v}_{L}, \quad v_{g}<\tilde{v}_{R}, \quad \tilde{v}_{d}>\bar{v}_{L} .
$$


This leads us to the characterization

$$
v_{g}<\bar{v}_{L}<v_{d} \leq \bar{v}_{R}
$$

and we obtain a solution consisting of a stationary discontinuity followed by a R-wave.

(g) For $\left(v_{g}<\bar{v}_{L}, v_{d}>\bar{v}_{R}\right)$, the conditions (34) become

$$
v_{d}>\bar{v}_{L}, \quad v_{g}<\bar{v}_{R}, \quad \bar{v}_{R}>\bar{v}_{L}
$$

and therefore

$$
v_{g}<\bar{v}_{L}<\bar{v}_{R}<v_{d}
$$

We find a solution consisting of a L-wave followed by a stationary discontinuity and a R-wave.

Observe that, in each case (d)-(g), the conditions (34) are exactly the conditions obtained in the previous section which ensure the existence and uniqueness of a v-discontinuous solution. We thus have proved

Theorem 1 Assume that the functions $f_{L}$ and $f_{R}$ are strictly convex and possess sonic states. Then the coupled Riemann problem has at least one solution. The solution is unique except in the case (c) where there exists a one-parameter family of v-continuous solutions.

We pass to the general case of arbitrary flux functions. The situation is not as simple as in the above examples due to the possible presence of several sonic states. The purpose of the remaining part of this section is to prove

Theorem 2 Assume that the flux functions $f_{L}$ and $f_{R}$ are $C^{1}$ functions. Then the coupled Riemann problem has at least one self-similar solution.

We know already that we can construct a v-continuous solution in the following cases:

$$
v_{d} \in \widetilde{\mathcal{F}}_{L}^{-}\left(v_{g}\right), \quad v_{g} \in \widetilde{\mathcal{F}}_{R}^{+}\left(v_{d}\right), \quad \widetilde{\mathcal{F}}_{L}^{-}\left(v_{g}\right) \cap \widetilde{\mathcal{F}}_{R}^{+}\left(v_{d}\right) \neq \emptyset .
$$

It remains to construct at least one v-discontinuous solution of the coupled Riemann problem when

$$
v_{d} \notin \widetilde{\mathcal{F}}_{L}^{-}\left(v_{g}\right), \quad v_{g} \notin \widetilde{\mathcal{F}}_{R}^{+}\left(v_{d}\right), \quad \widetilde{\mathcal{F}}_{L}^{-}\left(v_{g}\right) \cap \widetilde{\mathcal{F}}_{R}^{+}\left(v_{d}\right)=\emptyset .
$$

We begin with the following remarks. The condition $v_{d} \notin \widetilde{\mathcal{F}}_{L}^{-}\left(v_{g}\right)$ means that $z_{L}\left(\cdot ; v_{g}, v_{d}\right)$ possesses a nontrivial subwave whose speed is nonnegative. Otherwise, we would get $z_{L}\left(\frac{x}{t} ; v_{g}, v_{d}\right)=v_{d}$ for all $x \geq 0$ and therefore $v_{d} \in \widetilde{\mathcal{F}}_{L}^{-}\left(v_{g}\right)$. Hence, we have

$$
v_{L}\left(0_{-}\right) \stackrel{\text { def }}{=} z_{L}\left(0_{-} ; v_{g}, v_{d}\right) \neq v_{d} .
$$

Similarly, the condition $v_{g} \notin \widetilde{\mathcal{F}}_{R}^{+}\left(v_{d}\right)$ means that $z_{R}\left(\cdot ; v_{g}, v_{d}\right)$ possesses a nontrivial subwave whose speed is nonpositive so that

$$
v_{R}\left(0_{+}\right) \stackrel{\text { def }}{=} z_{R}\left(0_{+} ; v_{g}, v_{d}\right) \neq v_{g} .
$$

Note that the hypotheses (35) imply $v_{L}\left(0_{-}\right) \neq v_{R}\left(0_{+}\right)$. Otherwise the function

$$
z\left(\frac{x}{t} ; v_{g}, v_{d}\right)=\left\{\begin{array}{cc}
z_{L}\left(\frac{x}{t} ; v_{g}, v_{L}\left(0_{-}\right)\right), & \frac{x}{t}<0 \\
z_{R}\left(\frac{x}{t} ; v_{R}\left(0_{+}\right), v_{d}\right), & \frac{x}{t}>0
\end{array}\right.
$$


would be a v-continuous solution of the coupled Riemann problem. On the other hand, we have either $v_{L}\left(0_{-}\right)=v_{g}\left(\right.$ resp. $\left.v_{R}\left(0_{+}\right)=v_{d}\right)$ or $v_{L}\left(0_{-}\right)$(resp. $v_{R}\left(0_{+}\right)$) is a sonic state of $\tilde{f}_{L}$ (resp. $\tilde{f}_{R}$ ). Hence, it appears fairly natural to consider the function

$$
z\left(\frac{x}{t} ; v_{g}, v_{d}\right)= \begin{cases}z_{L}\left(\frac{x}{t} ; v_{g}, v_{d}\right), & x<0 \\ z_{R}\left(\frac{x}{t} ; v_{g}, v_{d}\right), & x>0\end{cases}
$$

as a possible solution of the coupled Riemann problem. Indeed, we can state

Lemma 9 Assume the hypotheses (35) together with

$$
\left\{\begin{array}{l}
v_{L}\left(0_{-}\right)<v_{R}\left(0_{+}\right) \quad \text { if } v_{g}<v_{d} \\
v_{L}\left(0_{-}\right)>v_{R}\left(0_{+}\right) \quad \text { if } v_{g}>v_{d} .
\end{array}\right.
$$

Then (36) is a solution of the coupled Riemann problem.

Proof. We have only to check the coupling conditions which read here

$$
\min _{v \in I\left(v_{L}\left(0_{-}\right), v_{R}\left(0_{+}\right)\right)} \frac{\tilde{f}_{L}(v)-\tilde{f}_{L}\left(v_{L}\left(0_{-}\right)\right)}{v-v_{L}\left(0_{-}\right)} \geq 0
$$

and

$$
\max _{v \in I\left(v_{L}\left(0_{-}\right), v_{R}\left(0_{+}\right)\right)} \frac{\tilde{f}_{R}(v)-\tilde{f}_{R}\left(v_{R}\left(0_{+}\right)\right)}{v-v_{R}\left(0_{+}\right)} \geq 0 .
$$

Assume for instance $v_{d}>v_{g}$. Since, in that case, $z_{L}\left(\cdot ; v_{g}, v_{d}\right)$ and $z_{R}\left(\cdot ; v_{g}, v_{d}\right)$ are monotonically increasing functions, we have by (37)

$$
v_{g} \leq v_{L}\left(0_{-}\right)<v_{R}\left(0_{+}\right) \leq v_{d} .
$$

Now, we observe that $z_{L}\left(\cdot ; v_{L}\left(0_{-}\right), v_{d}\right)$ has a nonnegative speed, i.e.,

$$
\min _{v \in I\left(v_{L}\left(0_{-}\right), v_{d}\right)} \frac{\tilde{f}_{L}(v)-\tilde{f}_{L}\left(v_{L}\left(0_{-}\right)\right)}{v-v_{L}\left(0_{-}\right)} \geq 0
$$

which implies the first coupling condition. On the other hand, $z_{R}\left(\cdot ; v_{g}, v_{R}\left(0_{+}\right)\right)$ has a nonpositive speed, i.e.,

$$
\max _{v \in I\left(v_{g}, v_{R}\left(0_{+}\right)\right.} \frac{\tilde{f}_{R}(v)-\tilde{f}_{R}\left(v_{R}\left(0_{+}\right)\right)}{v-v_{R}\left(0_{+}\right)} \leq 0
$$

which yields the second coupling condition. The case $v_{g}>v_{d}$ is analyzed in the same way.

Note that the proof of the above lemma only uses the first two hypotheses (35). Observe that this proof fails if the conditions (37) do not hold. It remains to construct a solution of the coupled Riemann problem when either

$$
v_{g}<v_{d} \quad \text { and } \quad v_{L}\left(0_{-}\right)>v_{R}\left(0_{+}\right)
$$




$$
v_{g}>v_{d} \text { and } v_{L}\left(0_{-}\right)<v_{R}\left(0_{+}\right) .
$$

Assume first (38). Let us then check that there exists at least one sonic state of $\tilde{f}_{L}$ in $\left[v_{g}, v_{R}\left(0_{+}\right)\right]$. It is here convenient to work with the conservative variable $u$ : setting $u_{g}=\theta_{L}\left(v_{g}\right), u_{R}\left(0_{+}\right)=\theta_{L}\left(v_{R}\left(0_{+}\right)\right)$, we introduce the lower convex envelope of $f_{L}$ in the interval $\left[u_{g}, u_{R}\left(0_{+}\right)\right]$. This envelope function cannot be strictly decreasing. Otherwise, $w_{L}\left(\cdot ; u_{g}, u_{R}\left(0_{+}\right)\right)$and therefore $z_{L}\left(\cdot ; v_{g}, v_{R}\left(0_{+}\right)\right)$, would be a wave whose speed is negative. One then could exhibit a v-continuous solution of the coupled Riemann problem, namely

$$
z\left(\frac{x}{t} ; v_{g}, v_{d}\right)= \begin{cases}z_{L}\left(\frac{x}{t} ; v_{g}, v_{R}\left(0_{+}\right)\right), & \frac{x}{t}<0 \\ z_{R}\left(\frac{x}{t} ; v_{R}\left(0_{+}\right), v_{d}\right), & \frac{x}{t}>0 .\end{cases}
$$

Hence the above envelope function has either a unique minimum which is a sonic state of $f_{L}$ or an interval of minima which contains such sonic states (at least the end points of this interval). Denote by $\bar{u}_{-}$the smallest of all sonic states of both $f_{L}$ and its lower convex envelope in $\left[u_{g}, u_{R}\left(0_{+}\right)\right]$. Then, $\bar{v}_{-}=\theta_{L}^{-1}\left(\bar{u}_{-}\right)$ is a sonic state of $\tilde{f}_{L}$ in $\left[v_{g}, v_{R}\left(0_{+}\right)\right]$. In the same way, there exists at least one sonic state of both $f_{R}$ and its lower convex envelope in the interval $\left[u_{L}\left(0_{-}\right), u_{d}\right]$ and we denote by $\bar{u}_{+}$the largest of all such sonic states. Then, $\bar{v}_{+}=\theta_{R}^{-1}\left(\bar{u}_{+}\right)$ is a sonic state of $\tilde{f}_{R}$ in $\left[v_{L}\left(0_{-}\right), v_{d}\right]$. Now, it appears natural to consider the functions

$$
z\left(\frac{x}{t} ; v_{g}, v_{d}\right)=\left\{\begin{array}{l}
z_{L}\left(\frac{x}{t} ; v_{g}, \bar{v}_{-}\right), \frac{x}{t}<0 \\
z_{R}\left(\frac{x}{t} ; v_{R}\left(0_{+}\right), v_{d}\right), \quad \frac{x}{t}>0
\end{array}\right.
$$

and

$$
z\left(\frac{x}{t} ; v_{g}, v_{d}\right)=\left\{\begin{array}{l}
z_{L}\left(\frac{x}{t} ; v_{g}, v_{L}\left(0_{-}\right)\right), \quad \frac{x}{t}<0 \\
z_{R}\left(\frac{x}{t} ; \bar{v}_{+}, v_{d}\right), \quad \frac{x}{t}>0
\end{array}\right.
$$

as possible candidates to the solution of the coupled Rieman problem. In fact, we can state

Lemma 10 Assume the hypotheses (35) and (38). Then (40) and (41) are solutions of the coupled Riemann problem.

Proof. Let us show that (40) is indeed solution. Again, we have to check the associated coupling conditions

$$
\bar{v}_{-}=z_{L}\left(0_{-} ; \bar{v}_{-}, v_{R}\left(0_{+}\right)\right) \Leftrightarrow \bar{u}_{-}=w_{L}\left(0_{-} ; \bar{u}_{-}, u_{R}\left(0_{+}\right)\right)
$$

and

$$
v_{R}\left(0_{+}\right)=z_{R}\left(0_{+} ; \bar{v}_{-}, v_{R}\left(0_{+}\right)\right) \Leftrightarrow u_{R}\left(0_{+}\right)=w_{R}\left(0_{+} ; \bar{u}_{-}, u_{R}\left(0_{+}\right)\right) .
$$

The first coupling condition holds since, by construction, $w_{L}\left(\cdot ; \bar{u}_{-}, u_{R}\left(0_{+}\right)\right)$is a monotonically increasing function in $\left[\bar{u}_{-}, u_{R}\left(0_{+}\right)\right]$and the corresponding wave has a nonnegative speed. Consider next the second coupling condition. We 


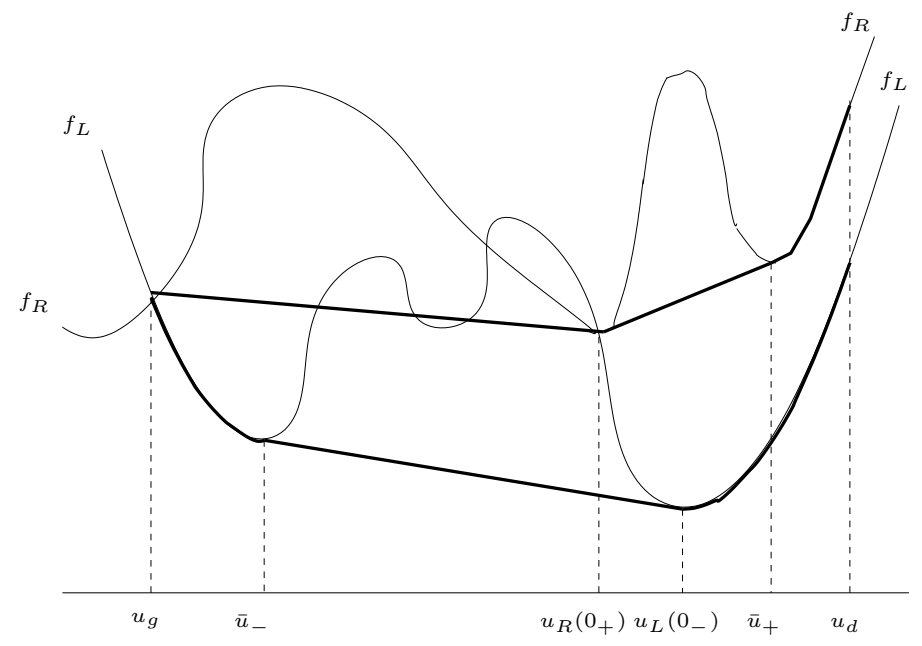

Figure 12: A typical example where $v_{d} \notin \widetilde{\mathcal{F}}_{L}^{-}\left(v_{g}\right), \quad v_{g} \notin \widetilde{\mathcal{F}}_{R}^{+}\left(v_{d}\right), \quad \widetilde{\mathcal{F}}_{L}^{-}\left(v_{g}\right) \cap$ $\widetilde{\mathcal{F}}_{R}^{+}\left(v_{d}\right)=\emptyset$.

know that the lower convex envelope of $f_{R}$ in $\left[u_{g}, u_{d}\right]$ is a monotonically decreasing function in the interval $\left[u_{g}, u_{R}\left(0_{+}\right)\right]$and is strictly convex in an interval $\left[u_{R}\left(0_{+}\right), u_{R}\left(0_{+}\right)+\varepsilon\right], \varepsilon>0$ small enough (cf. Fig. 12). Then, as $u_{g}<\bar{u}_{-}<u_{R}\left(0_{+}\right)$, it is clear geometrically that the lower convex envelope of $f_{R}$ in the interval $\left[\bar{u}_{-}, u_{R}\left(0_{+}\right)\right]$is a monotonically decreasing function so that $u_{R}\left(0_{+}\right)=w_{R}\left(0_{+} ; \bar{u}_{-}, u_{R}\left(0_{+}\right)\right)$and our assertion is proved.

By using similar arguments, one can prove that (41) is also solution.

Remark. At first glance it would seem natural to consider the function

$$
z\left(\frac{x}{t} ; v_{g}, v_{d}\right)=\left\{\begin{array}{cc}
z_{L}\left(\frac{x}{t} ; v_{g}, \bar{v}_{-}\right), & \frac{x}{t}<0 \\
z_{R}\left(\frac{x}{t} ; \bar{v}_{+}, v_{d}\right), & \frac{x}{t}>0
\end{array}\right.
$$

as a possible solution of the coupled Riemann problem. However, this is not true since one can easily check that the coupling conditions

$$
\bar{v}_{-}=z_{L}\left(0_{-} ; \bar{v}_{-}, \bar{v}_{+}\right), \quad \bar{v}_{+}=z_{R}\left(0_{+} ; \bar{v}_{-}, \bar{v}_{+}\right)
$$

are not satisfied in general (cf. Fig. 12).

We can also state the analogue of Lemma 10 whose proof follows the same lines as above.

Lemma 11 Assume the hypotheses (35) and (39). Then the coupled Riemann problem has at least two v-discontinuous solutions.

Theorem 3 is now an obvious consequence of Lemmas 10 and 11 .

A natural question now arises: when the coupled Riemann problem posesses several solutions, does there exist any "reasonable" criterion based on entropy or stability arguments for choosing the "right solution"? As a first step in this direction, we conjecture that, if a v-continuous solution exists, the eventual $\mathrm{v}$-discontinuous solutions should be considered as parasitic ones. 


\subsection{The coupled Riemann problem for two conservation laws "with phase change".}

One can extend the above results to the case where the flux functions $f_{\alpha}, \alpha=$ $L, R$, are only piecewise $C^{1}$. For simplicity, we will restrict ourselves in this section to continuous functions $f_{\alpha}$ which satisfy the following properties:

(i) $f_{\alpha}$ is a $C^{1}$ strictly increasing function in the intervals $\left(-\infty, a_{\alpha}\right)$ and $\left(b_{\alpha},+\infty\right)$, $a_{\alpha}<b_{\alpha}$;

(ii) $f_{\alpha}$ is constant in the interval $\left[a_{\alpha}, b_{\alpha}\right]$.

One can think of each flux function $f_{\alpha}$ as modeling a diphasic behavior: the states $u<a_{\alpha}$ and $u>b_{\alpha}$ correspond to different phases while the states $u \in$ $\left[a_{\alpha}, b_{\alpha}\right]$ correspond to a mixture of the two phases.

Again for simplicity, we will restrict ourselves to the u-coupling method. Before constructing the solution of the coupled Riemann problem, let us recall the properties of the solution $w\left(\cdot ; u_{g}, u_{d}\right)$ of the usual Riemann problem associated with such a function $f=f_{\alpha}{ }^{3}$. By introducing the lower convex envelope (resp. the upper concave envelope) of $f$ between the states $u_{g}$ and $u_{d}$ if $u_{g}<u_{d}$ (resp. $\left.u_{g}>u_{d}\right)$, it is a simple matter to check the following properties of $w\left(\cdot ; u_{g}, u_{d}\right)$ :

(i) the associated (composite) wave has a nonnegative speed;

(ii) the function $x \rightarrow w\left(\frac{x}{t} ; u_{g}, u_{d}\right)$ is continuous at $x=0$ in the following cases

$$
\begin{cases}u_{g}<a, & u_{d} \in \mathbb{R} \\ u_{g}=a, & u_{d}<a \\ u_{g}>b, & u_{d} \in \mathbb{R} \\ u_{g}=b, & u_{d}>b\end{cases}
$$

(iii) the function $x \rightarrow w\left(\frac{x}{t} ; u_{g}, u_{d}\right)$ is discontinuous at $x=0$ in the following cases

$$
\left\{\begin{array}{l}
u_{g}, u_{d} \in[a, b] \\
u_{g} \in[a, b), \quad u_{d}>b \\
u_{g} \in(a, b], \quad u_{d}<a .
\end{array}\right.
$$

In the first case, $w\left(\cdot ; u_{g}, u_{d}\right)$ consists of a stationary shock while, in the last two cases, $w\left(\cdot ; u_{g}, u_{d}\right)$ is a composite wave whose left subwave is a stationary shock.

Let us now consider the coupled Riemann problem. Instead of establishing general results for piecewise $C^{1}$ flux functions, it is here far simpler to use a direct approach. Since the function $f_{L}$ is monotonically increasing, the solution of the coupled Riemann problem cannot include a L-wave. Therefore a solution consists of a possible stationary shock wave connecting $u_{g}$ and $u_{+}$and a R-wave connecting $u_{+}$and $u_{d}$.

Assume first $u_{g}=u_{+}$so that the solution is continuous at the interface $x=0$. Then, using (42), we know that this is indeed the case if and only if the pair $\left(u_{g}, u_{d}\right)$ satisfies one of the following properties

$$
\begin{cases}u_{g}<a_{R}, & u_{d} \in \mathbb{R} \\ u_{g}=a_{R}, & u_{d}<a_{R} \\ u_{g}>b_{R}, & u_{d} \in \mathbb{R} \\ u_{g}=b_{R}, & u_{d}>b_{R} .\end{cases}
$$

Assume next $u_{g} \neq u_{+}$. This occurs if and only if, on the one hand, the coupling conditions hold and, on the other hand, $w_{R}\left(\cdot ; u_{+}, u_{d}\right)$ is either a trivial wave

\footnotetext{
${ }^{3}$ we drop the subscript $\alpha$ for simplicity.
} 
(i.e., $u_{d}=u_{+}$) or a wave whose speed is positive. Since the function $f_{L}$ is monotonically increasing, the first coupling condition

$$
\min _{u \in I\left(u_{g}, u_{+}\right)} \frac{f_{L}(u)-f_{L}\left(u_{g}\right)}{u-u_{g}} \geq 0
$$

holds trivially. The second coupling condition

$$
\max _{u \in I\left(u_{g}, u_{+}\right)} \frac{f_{R}(u)-f_{R}\left(u_{+}\right)}{u-u_{+}} \leq 0
$$

means that the wave $w_{R}\left(\cdot ; u_{g}, u_{+}\right)$has a nonpositive speed. Hence $w_{R}\left(\cdot ; u_{g}, u_{+}\right)$ is necessarily a stationary shock or equivalently (cf. property (iii) above) we have

$$
u_{g}, u_{+} \in\left[a_{R}, b_{R}\right] .
$$

If $u_{+}=u_{d}$, we thus have an admissible stationary shock for the solution of the coupled Riemann problem as soon as

$$
u_{g}, u_{d} \in\left[a_{R}, b_{R}\right], \quad u_{g} \neq u_{d} .
$$

Consider next the case $u_{+} \neq u_{d}$. For the speed of the wave $w_{R}\left(\cdot ; u_{+}, u_{d}\right)$ to be positive, it follows from (42) that we must have either

$$
u_{+}=a_{R}, \quad u_{d}<a_{R}
$$

or

$$
u_{+}=b_{R}, \quad u_{d}>b_{R} .
$$

In both cases, one can easily check that the speed of the wave is indeed positive.

As a conclusion, we obtain that the coupled Riemann problem has a unique solution. This solution is u-continuous at the interface $x=0$ in the cases (44) and is u-discontinuous otherwise, i.e., when either

$$
u_{g}, u_{d}=u_{+} \in\left[a_{R}, b_{R}\right], \quad u_{g} \neq u_{d}
$$

or

$$
\left\{\begin{array}{lll}
u_{g} \in\left(a_{R}, b_{R}\right], & u_{+}=a_{R}, & u_{d}<a_{R} \\
u_{g} \in\left[a_{R}, b_{R}\right), & u_{+}=b_{R}, & u_{d}>b_{R} .
\end{array}\right.
$$

This result is easily extended to the case of a v-coupling method. It may be viewed as a generalization of the results of Example 2.3 when both flux functions $f_{\alpha}$ are strictly increasing.

\section{$3 \quad$ Numerical experiments}

Our objective in this section is to illustrate numerically the theoretical results we obtained in the previous sections. For that, the following configurations will be considered :

- the case of two strictly monotone flux functions (example 2.3 above),

- the case of two strictly convex flux functions (example 2.4 above), 
- a particular configuration where two discontinuous and none continuous (at the coupling interface) solutions are admissible,

- a particular configuration where several discontinuous solutions and continuous solutions exist,

- and the coupling of two conservation laws "with phase change".

The situations leading to several admissible solutions (continuous or discontinuous at interface) are of particular interest since different numerical schemes may capture different solutions. We begin with a brief description of the proposed numerical strategy and then present some numerical results.

\subsection{Numerical strategy}

We consider a finite volume approach. Let $\Delta x$ and $\Delta t$ denote the uniform space and time steps and $\mathcal{C}_{j+1 / 2}$ be the cells defined by $\mathcal{C}_{j+1 / 2}=\left(x_{j}, x_{j+1}\right)$ with $x_{j}=j \Delta x$ and whose centers are $x_{j+1 / 2}=(j+1 / 2) \Delta x$ for all $j \in \mathbb{Z}$. We set $\lambda=\Delta t / \Delta x$ and $t_{n}=n \Delta t$ for $n \in \mathbb{N}$. The approximate solution is assumed to be piecewise constant on each cell $\mathcal{C}_{j+1 / 2}$ and at each time $t^{n}$ and the corresponding value is denoted $u_{j+1 / 2}^{n}$.

To begin with, we set as usual

$$
u_{j+1 / 2}^{0}=\frac{1}{\Delta x} \int_{\mathcal{C}_{j+1 / 2}} u_{0}(x) d x, \quad j \in \mathbb{Z},
$$

where $u_{0}$ denotes a given initial condition of the coupling problem.

Then, let $G_{\alpha}, \alpha=L, R$ be two two-point numerical flux functions that we assume to be consistant with $f_{\alpha}, \alpha=L, R$. We propose the following update formula for $u_{j+1 / 2}^{n+1}$ :

$$
\begin{array}{ll}
u_{j-1 / 2}^{n+1}=u_{j-1 / 2}^{n}-\lambda\left(G_{L, j}^{n}-G_{L, j-1}^{n}\right), & j \leq 0, n \geq 0, \\
u_{j+1 / 2}^{n+1}=u_{j+1 / 2}^{n}-\lambda\left(G_{R, j+1}^{n}-G_{R, j}^{n}\right), & j \geq 0, n \geq 0,
\end{array}
$$

with $G_{\alpha, j}^{n}=G_{\alpha}\left(u_{j-1 / 2}^{n}, u_{j+1 / 2}^{n}\right)$ for $j \neq 0$. In other words, this consists in a classical finite volume scheme outside of the interface, and only both fluxes $G_{L, 0}^{n}$ and $G_{R, 0}^{n}$ remain to be precised in order to define the numerical coupling procedure at the interface. Following the previous works [19], [18], [3] (see also [4], [5]), we set

$$
\begin{aligned}
& G_{L, 0}^{n}=G_{L}\left(u_{-1 / 2}^{n}, \theta_{L}\left(v_{1 / 2}^{n}\right)\right), \\
& G_{R, 0}^{n}=G_{R}\left(\theta_{R}\left(v_{-1 / 2}^{n}\right), u_{1 / 2}^{n}\right),
\end{aligned}
$$

where ghost states $v_{ \pm 1 / 2}^{n}$ are obtained as

$$
\begin{aligned}
v_{-1 / 2}^{n} & =\theta_{L}^{-1}\left(u_{-1 / 2}^{n}\right), \\
v_{1 / 2}^{n} & =\theta_{R}^{-1}\left(u_{1 / 2}^{n}\right) .
\end{aligned}
$$

Note from now on that for convenience, we will restrict ourselves to the simple case $\theta_{L}=\theta_{R}=i d$, so that condition (5) reads $u\left(t, 0^{-}\right)=u\left(t, 0^{+}\right)$and the ghost states at the interface are simply

$$
\begin{aligned}
v_{-1 / 2}^{n} & =u_{-1 / 2}^{n}, \\
v_{1 / 2}^{n} & =u_{1 / 2}^{n} .
\end{aligned}
$$


At last and as far as the numerical flux functions $G_{\alpha}, \alpha=L, R$ are concerned, we will consider the celebrated Godunov scheme :

$$
G_{\alpha}(u, v)= \begin{cases}\min _{w \in[u, v]} f_{\alpha}(w), & u \leq v, \\ \max _{w \in[v, u]} f_{\alpha}(w), & v<u,\end{cases}
$$

and a relaxation scheme (see for instance [25]) defined by :

$G_{\alpha}(u, v)=\frac{1}{2}\left(f_{\alpha}(u)+f_{\alpha}(v)\right)+\frac{a(u, v)}{2}(u-v) \quad$ with $\quad a(u, v)=\max _{[\min (u, v), \max (u, v)]}\left|f^{\prime}\right|$.

\subsection{Numerical results}

Let us now present the numerical tests and results.

Test 1. The case of strictly monotone flux functions.

As Riemann initial data, we take

$$
u_{0}(x)=\left\{\begin{array}{lll}
u_{g} & \text { if } \quad x<0, \\
u_{d} & \text { if } \quad x>0,
\end{array}\right.
$$

with $u_{g}=-2$ and $u_{d}=2$ and we consider the following cases :

(a) $f_{L}(u)=-u$ and $f_{R}(u)=-2 u$ : the unique solution is continuous at the coupling interface.

(b) $f_{L}(u)=u$ and $f_{R}(u)=2 u$ : the unique solution is continuous at the coupling interface.

(c) $f_{L}(u)=u$ and $f_{R}(u)=-2 u$ : there is no continuous solution but a unique discontinuous solution.

(d) $f_{L}(u)=-u$ and $f_{R}(u)=2 u$ : there is no discontinuous solution and a continuum of continuous solutions.

Numerical results, obtained for both relaxation and Godunov approaches, are presented on Fig.13. These results are in agreement with the above theoretical results. Note that in the last case both numerical schemes capture the same continuous solution.

Test 2. The case of strictly convex flux functions with sonic states.

We consider two different cases, according to the relative position of the sonic points $\bar{v}_{L}$ and $\bar{v}_{R}$.

Test 2.1. $f_{L}(u)=u^{2} / 2, f_{R}(u)=(u-1)^{2} / 2$.

The sonic points are $\bar{v}_{L}=0$ and $\bar{v}_{R}=1$ and we consider the following four Riemann problems :

(a) $u_{g}=-1$ and $u_{d}=2$ : the unique solution is a discontinuous solution of the fourth type (composite wave consisting of a L-wave, a discontinuity and a R-wave).

(b) $u_{g}=2$ and $u_{d}=-1$ : the unique solution is a discontinuous solution of the first type. 


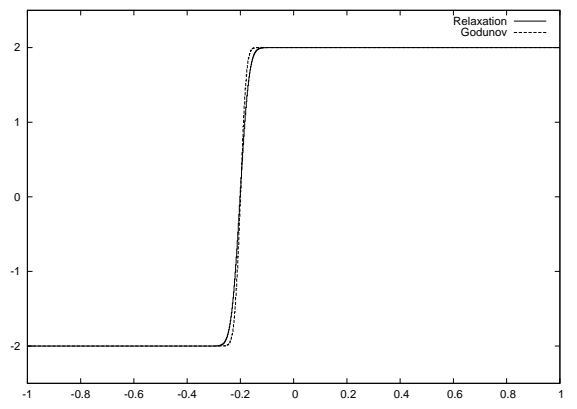

(a)

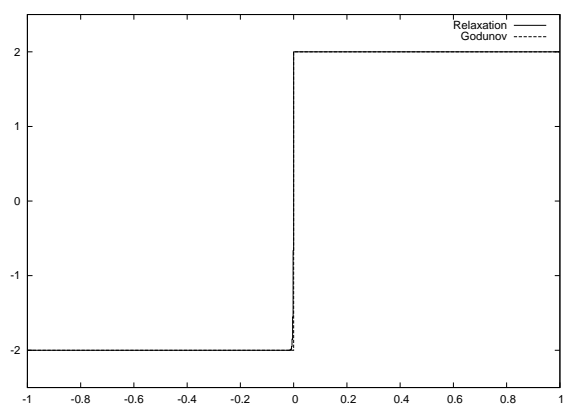

(c)

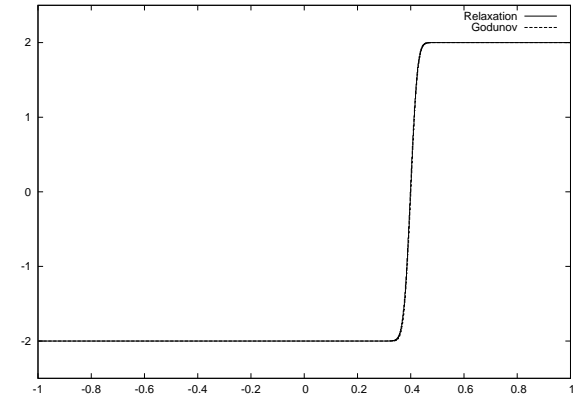

(b)

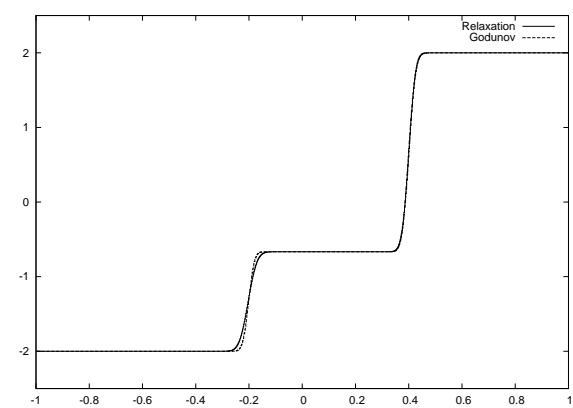

(d)

Figure 13: Monotone fluxes. 1000 pts. $t=0.2 . u_{g}=-2, u_{d}=2$.

(c) $u_{g}=0.5$ and $u_{d}=-2$ : the solutions are a continuous solution of the first type and a discontinuous solution of the first type.

(d) $u_{g}=-1$ and $u_{d}=-1.5$ : the solutions are a continuous solution of the first type and a discontinuous solution of the second type (that is not a monotonous solution).

Numerical solutions are presented on Fig.14. We observe that both numerical schemes select the continuous solutions when more are presents (cases (c) and (d)).

Test 2.2. $f_{L}(u)=u^{2} / 2, f_{R}(u)=(u+1)^{2} / 2$.

The sonic points are $\bar{v}_{L}=0$ and $\bar{v}_{R}=-1$ and we consider the following four Riemann problems. The numerical solutions are presented on Fig.15.

(a) $u_{g}=-0.6$ and $u_{d}=-0.2$ : the solutions are a continuous solution of the first, second or third type, and a discontinuous solution of the fourth type. Both numerical schemes capture the continuous solution of the third type, here with two rarefaction waves connecting a constant state, that slightly differs for both schemes.

(b) $u_{g}=-0.2$ and $u_{d}=-0.6$ : the solutions are a continuous solution of the first, second or third type, and a discontinuous solution of the fourth type. Both numerical schemes capture the continuous solution of the third type, 


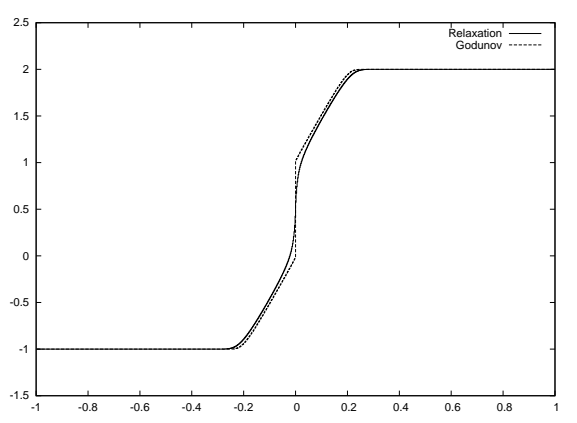

(a) $u_{g}=-1, u_{d}=2$

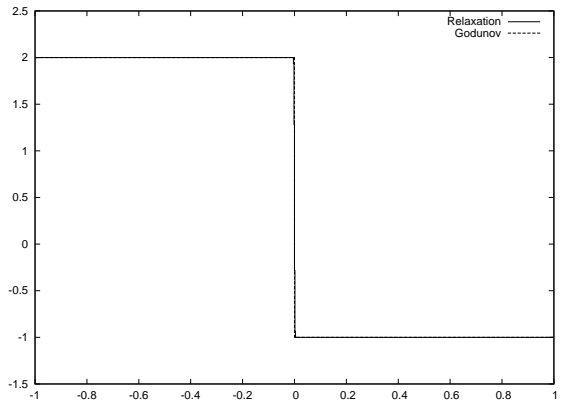

(b) $u_{g}=2, u_{d}=-1$

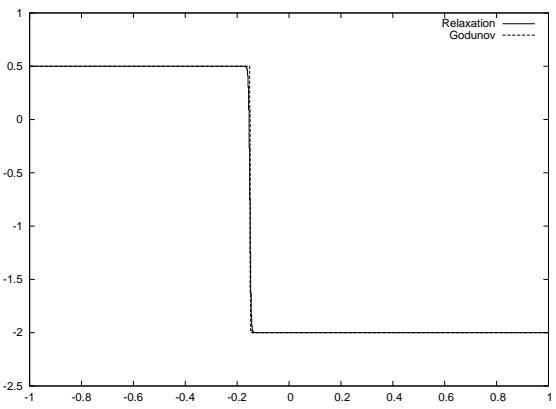

(c) $u_{g}=0.5, u_{d}=-2$

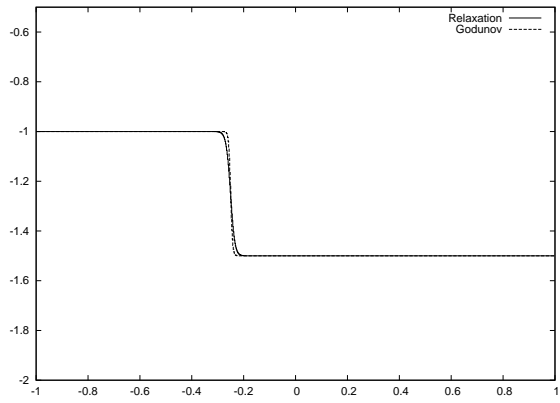

(d) $u_{g}=-1, u_{d}=-1.5$

Figure 14: Convex fluxes. 1000 pts. $t=0.2$

here with two shock waves connecting a constant state, that slightly differs for both schemes.

(c) $u_{g}=0.5$ and $u_{d}=1$ : the solutions are a continuous solution of the second type, and a discontinuous solution of the third type. Both numerical schemes capture the unique continuous solution of the second type.

(d) $u_{g}=1$ and $u_{d}=-1.5$ : the solutions are a continuous solution of the first, second or third type, and a discontinuous solution of the first type. Both numerical schemes capture the continuous solution of the second type.

Test 3. A particular configuration where only two discontinuous solutions are admissible. 


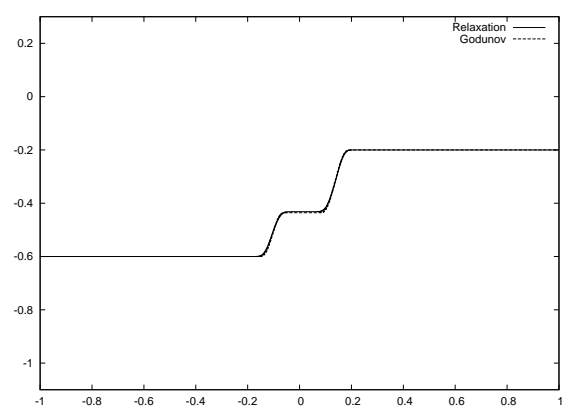

(a) $u_{g}=-0.6, u_{d}=-0.2$

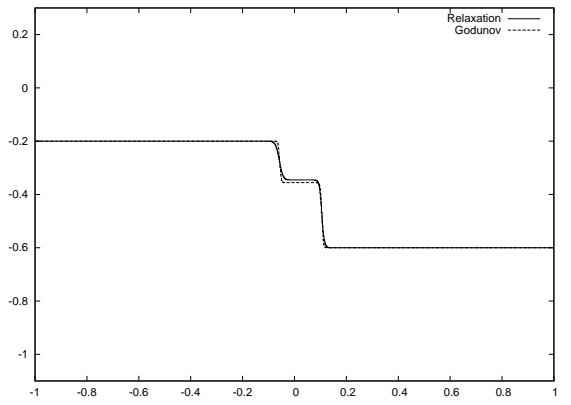

(b) $u_{g}=-0.2, u_{d}=-0.6$

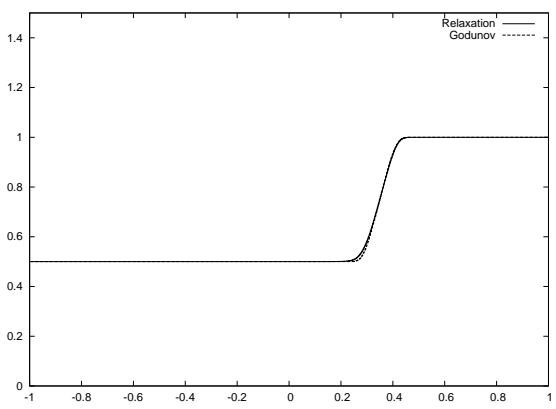

(c) $u_{g}=0.5, u_{d}=1$

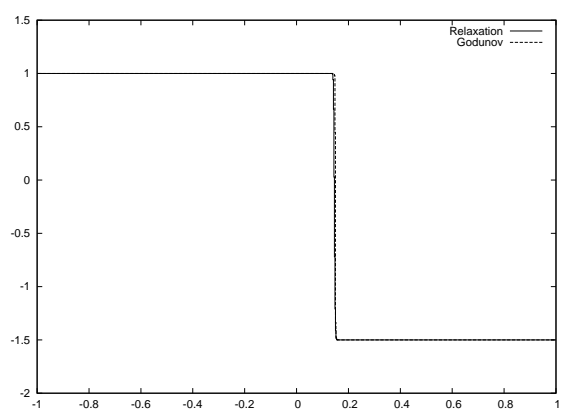

(d) $u_{g}=1, u_{d}=-1.5$

Figure 15: Convex fluxes. 1000 pts. $t=0.2$

The flux functions $f_{L}$ and $f_{R}$ are defined from the derivatives $f_{L}^{\prime}$ and $f_{R}^{\prime}$ given by

$$
\begin{gathered}
f_{L}^{\prime}(u)=(u+1)\left(u+\frac{1}{10}\right)(u-1), \\
f_{R}^{\prime}(u)=-\left(u+\frac{1}{2}\right)\left(u-\frac{2}{5}\right)\left(u-\frac{3}{2}\right) .
\end{gathered}
$$

The sonic points are thus $u_{L}^{-}=-1, u_{L}^{0}=-1 / 10$ and $u_{L}^{+}=1$ for the left flux $f_{L}$ and $u_{R}^{-}=-1 / 2, u_{R}^{0}=2 / 5$ and $u_{R}^{+}=3 / 2$ for the right flux $f_{R}$. The Riemann initial data is such that $u_{g}=-1.25$ and $u_{d}=1.75$. This configuration is such that $u_{g} \notin \widetilde{\mathcal{F}}_{R}^{+}\left(u_{d}\right), u_{d} \notin \widetilde{\mathcal{F}}_{L}^{-}\left(u_{g}\right)$. Moreover the set $\widetilde{\mathcal{F}}_{L}^{-}\left(u_{g}\right) \cap \widetilde{\mathcal{F}}_{R}^{+}\left(u_{d}\right)$ is empty and therefore there is no continuous solution. Fig. 16 represents both fluxes 
and in bold-face the sets $\widetilde{\mathcal{F}}_{R}^{+}\left(u_{d}\right)$ and $\widetilde{\mathcal{F}}_{L}^{-}\left(u_{g}\right)$.

The proposed coupled Riemann problem admits only discontinuous solutions,

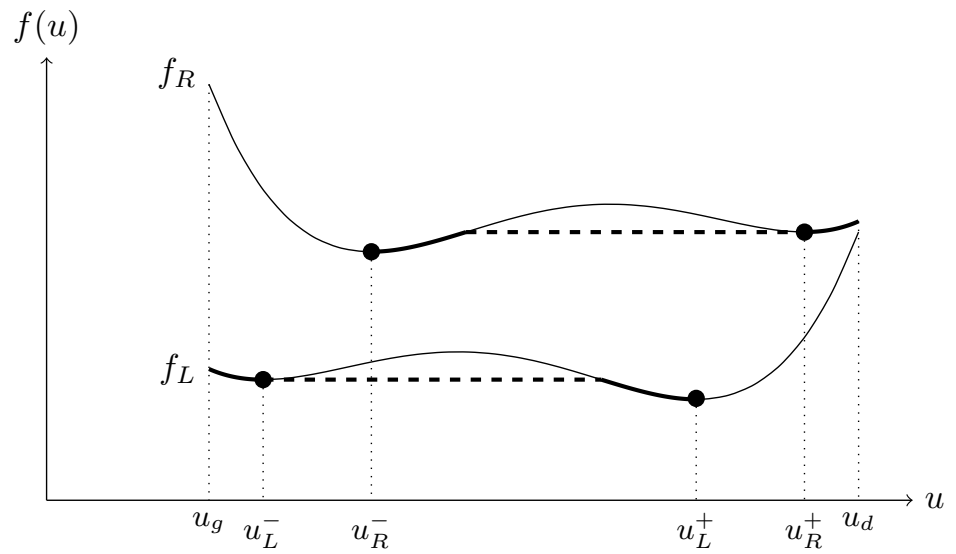

Figure 16: A case where $u_{g} \notin \widetilde{\mathcal{F}}_{R}^{+}\left(u_{d}\right), u_{d} \notin \widetilde{\mathcal{F}}_{L}^{-}\left(u_{g}\right)$, and $\widetilde{\mathcal{F}}_{R}^{+}\left(u_{d}\right) \cap \widetilde{\mathcal{F}}_{L}^{-}\left(u_{g}\right)=\emptyset$.

each one of the third type. More precisely, they are

(a) a L-wave (whose speed is nonpositive) connecting $u_{g}$ to $u_{L}^{+}$, followed by a $\mathrm{R}$-wave (whose speed is nonnegative) connecting $u_{R}^{+}$to $u_{d}$,

(b) a L-wave (whose speed is nonpositive) connecting $u_{g}$ to $u_{L}^{-}$, followed by a $\mathrm{R}$-wave (whose speed is nonnegative) connecting $u_{R}^{-}$to $u_{d}$.

Numerical solutions are presented on Fig. 17. We observe that the Godunov scheme captures the solution (b), while the relaxation scheme captures the solution (a).

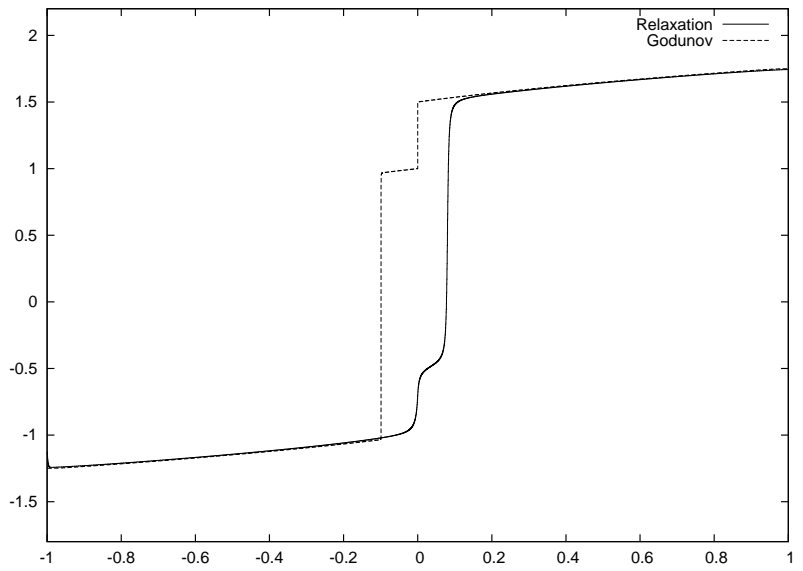

Figure 17: Multiple disc. solutions. 10000 pts. $u_{L}=-1.25, u_{R}=1.75, t=1.5$

Test 4. A particular configuration where several discontinuous solutions are admissible, and continuous solutions also exist. 
Here again, both fluxes are obtained from the derivatives given by

$$
\begin{gathered}
f_{L}^{\prime}(u)=(u+1)\left(u+\frac{1}{2}\right)(u-1), \\
f_{R}^{\prime}(u)=-\left(u-\frac{5}{4}\right)\left(u-\frac{3}{4}\right)\left(u+\frac{3}{4}\right) .
\end{gathered}
$$

We take $u_{g}=-1.5$ and $u_{d}=1.75$ so that $u_{g} \notin \widetilde{\mathcal{F}}_{R}^{+}\left(u_{d}\right), u_{d} \notin \widetilde{\mathcal{F}}_{L}^{-}\left(u_{g}\right)$, but the set $\widetilde{\mathcal{F}}_{L}^{-}\left(u_{g}\right) \cap \widetilde{\mathcal{F}}_{R}^{+}\left(u_{d}\right)$ is not empty. Fig. 18 represents both fluxes and in bold-face the sets $\widetilde{\mathcal{F}}_{R}^{+}\left(u_{d}\right)$ and $\widetilde{\mathcal{F}}_{L}^{-}\left(u_{g}\right)$.

We can see that both numerical schemes capture the same continuous solution.

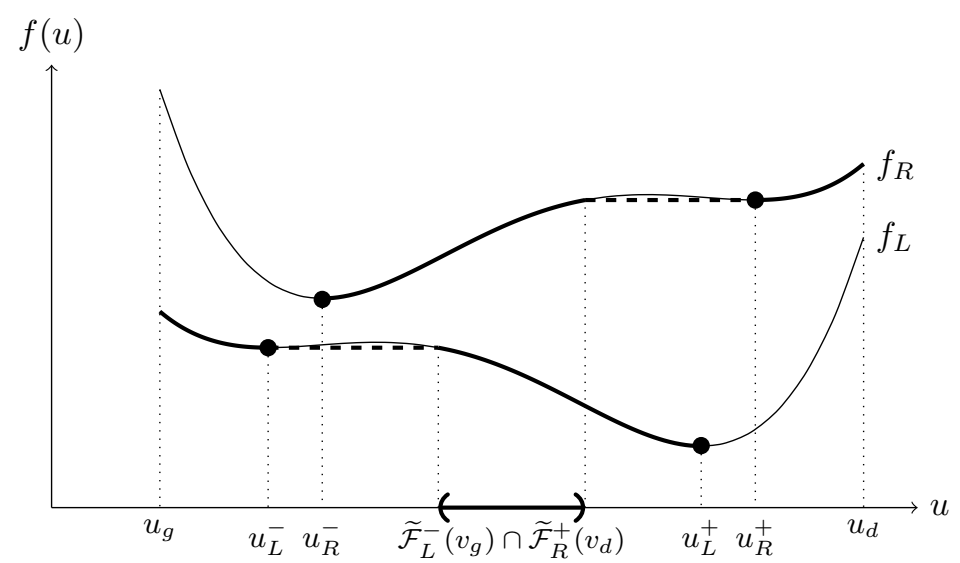

Figure 18: A case where $u_{g} \notin \widetilde{\mathcal{F}}_{R}^{+}\left(u_{d}\right), u_{d} \notin \widetilde{\mathcal{F}}_{L}^{-}\left(u_{g}\right)$, and $\widetilde{\mathcal{F}}_{R}^{+}\left(u_{d}\right) \cap \widetilde{\mathcal{F}}_{L}^{-}\left(u_{g}\right) \neq \emptyset$.

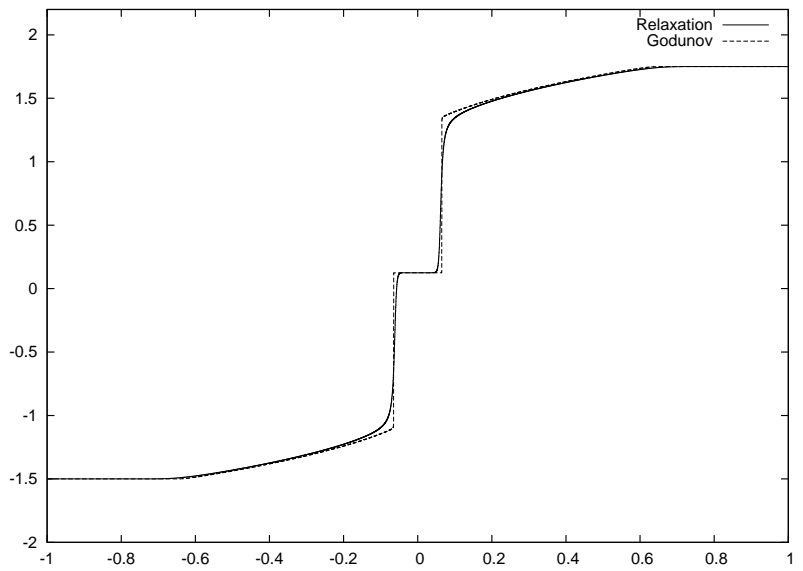

Figure 19: Multiple disc. solutions. 10000 pts. $t=0.5 . u_{L}=-1.5, u_{R}=1.75$.

Test 5. The coupling of two conservation laws "with phase change"

The rest of this section is devoted to the coupling of two scalar conservation 
laws "with phase change". We consider the following fluxes

$$
f_{L}(u)=\left\{\begin{array}{ll}
-\frac{u^{2}-1}{2}, & u<-1, \\
0, & -1 \leq u \leq 1, \\
\frac{u^{2}-1}{2}, & u>1,
\end{array} \quad f_{R}(u)=f_{L}(u-1 / 2)-1,\right.
$$

that are represented on Fig. 20. The numerical solutions are shown on Fig.21

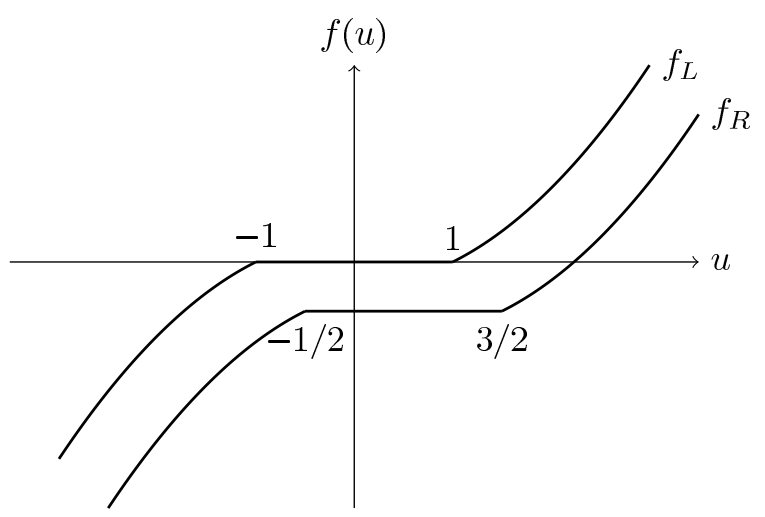

Figure 20: Scalar flux for the coupling "with phase change".

for both relaxation and Godunov schemes and these match with the expected theoretical results. They correspond to the solution for the right-problem, that consists here in a shock transition wave connecting the left-state of the Riemann problem to the point of $C^{1}$-discontinuity $(u=3 / 2)$, a constant part and finally a rarefaction wave to the right-state.

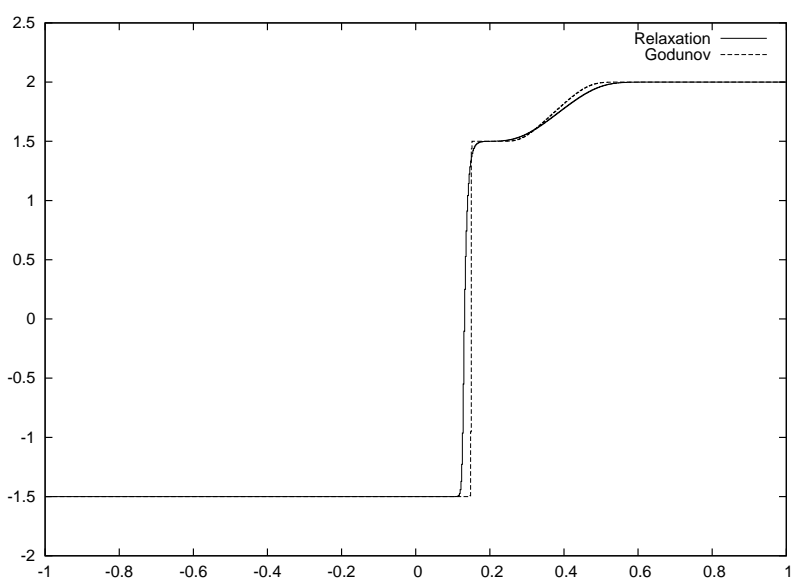

Figure 21: Phase change. 1000 pts. $u_{L}=-1.5, u_{R}=2.0, t=0.3$. 


\section{References}

[1] Adimurthi, A. Mishra, G.D. Veerappa Gowda. Convergence of Godunov type methods for a conservation law with a spatially varying discontinuous flux function. Math. Comp., 76, pp 1219-1242, 2007.

[2] A. Ambroso, C. Chalons, F. Coquel, T. Galié. Relaxation and numerical approximation of a two fluid two pressure diphasic model. Mathematical Modelling and Numerical Analysis (M2AN), vol 43, pp 1063-1097, 2009.

[3] A. Ambroso, C. Chalons, F. Coquel, E. Godlewski, F. Lagoutière, P.-A. Raviart, N. Seguin. The coupling of homogeneous models for two-phase flows. Int. Journal for Finite Volume, 4(1), 2007.

[4] A. Ambroso, C. Chalons, F. Coquel, E. Godlewski, F. Lagoutière, P.-A. Raviart, N. Seguin. Coupling of general Lagrangian systems. Mathematics of Computation, 77, pp 909-941, 2008.

[5] A. Ambroso, C. Chalons, F. Coquel, E. Godlewski, F. Lagoutière, P.-A. Raviart and N. Seguin. Relaxation methods and coupling procedure. International Journal for Numerical Methods in Fluids, 56(8):1123-1129, 2008.

[6] A. Ambroso, C. Chalons, F. Coquel, T. Galié, E. Godlewski, P.-A. Raviart and N. Seguin. The drift-flux asymptotic limit of barotropic two-phase twopressure models. Comm. Math. Sci., 6:521-529, 2008.

[7] F. Bachmann and J. Vovelle. Existence and uniqueness of entropy solution of scalar conservation law with a flux function involving discontinuous coefficients. Comm. Partial Differential Equations, 31:371-395, 2006.

[8] M. K. Banda, M. Herty, and A. Klar. Coupling conditions for gas networks governed by the isothermal Euler equations. Netw. Heterog. Media, 1(2):295314 (electronic), 2006.

[9] M. K. Banda, M. Herty, and A. Klar. Gas flow in pipeline networks. Netw. Heterog. Media, 1(1):41-56 (electronic), 2006.

[10] F. Caetano. Sur certains problèmes de linéarisation et de couplage pour les systèmes hyperboliques non linéaires. PhD thesis, Université Pierre et Marie Curie-Paris6, France, 2006.

[11] C. Chalons, P.-A. Raviart, N. Seguin. The interface coupling of the gas dynamics equations. Quarterly of Applied Mathematics, 66, no. 4, pp 659$705,2008$.

[12] R. M. Colombo and M. Garavello. A well posed Riemann problem for the p-system at a junction. Netw. Heterog. Media, 1(3):495-511 (electronic), 2006.

[13] F. Dubois and P.-G. LeFloch. Boundary conditions for nonlinear hyperbolic systems of conservation laws. J. Differential Equations, 71(1):93-122, 1988.

[14] T. Galié. Couplage interfacial de modèles en dynamique des fluides. Application aux écoulements diphasiques. PhD thesis, Université Pierre et Marie Curie - Paris 6, France, 2009. 
[15] M. Garavello, R. Natalini, B. Piccoli and A. Terracina. Conservation laws with discontinuous flux. Netw. Heterog. Media, 2(1):159-179, 2007.

[16] M. Garavello and B. Piccoli. Traffic flow in networks. AIMS book series, 2006.

[17] M. Garavello and B. Piccoli. Traffic flow on a road network using the Aw-Rascle model. Comm. Partial Differential Equations, 31(1-3):243-275, 2006.

[18] E. Godlewski, K.-C. Le Thanh and P.-A. Raviart. The numerical interface coupling of nonlinear hyperbolic systems of conservation laws. II. The case of systems. M2AN Math. Model. Numer. Anal., 39(4):649-692, 2005.

[19] E. Godlewski and P.-A. Raviart. The numerical interface coupling of nonlinear hyperbolic systems of conservation laws. I. The scalar case. Numer. Math., 97(1):81-130, 2004.

[20] A. Guelfi, D. Bestion, M. Boucker, P. Boudier, P. Fillion, M. Grandotto, J.M. Hérard, E. Hervieu, P. Péturaud. NEPTUNE: A New Software Platform for Advanced Nuclear Thermal Hydraulic. Nuclear Science and Engineering, 156, pp 281-324 (2007).

[21] M. Herty and M. Rascle. Coupling conditions for a class of second-order models for traffic flow. SIAM J. Math. Anal., 38(2):595-616 (electronic), 2006.

[22] O. Hurisse. Techniques de couplage de modèles hyperboliques en thermohydraulique diphasique. $\mathrm{PhD}$ thesis, Université de Provence, France, 2006.

[23] E. Isaacson and B. Temple. Nonlinear resonance in systems of conservation laws. SIAM J. Appl. Math., 52(5):1260-1278, 1992.

[24] C. Klingenberg and N. H. Risebro. Convex conservation laws with discontinuous coefficients. Existence, uniqueness and asymptotic behavior. Comm. Partial Differential Equations, 20(11-12):1959-1990, 1995.

[25] C. Lattanzio and D. Serre. Convergence of a relaxation scheme for hyperbolic systems of conservation laws. Numer. Math., vol 88, pp 121-134 (2001).

Benjamin Boutin

UMR 7598 Laboratoire Jacques-Louis Lions,

Université Pierre et Marie Curie-Paris6,

Boîte courrier 187, 75252 PARIS CEDEX 05, France

and

CEA-Saclay

DEN/DANS/DM2S/SFME/LETR

91191 Gif-sur-Yvette cedex, France

boutin@ann.jussieu.fr

Christophe Chalons

UMR 7598 Laboratoire Jacques-Louis Lions, 
Université Paris 7-Denis Diderot,

Boîte courrier 187, 75252 PARIS CEDEX 05, France and

CEA-Saclay

DEN/DANS/DM2S/SFME/LETR

91191 Gif-sur-Yvette cedex, France

chalons@math . jussieu .fr

Pierre-Arnaud RAVIART

UMR 7598 Laboratoire Jacques-Louis Lions,

CNRS \& Université Pierre et Marie Curie-Paris6,

Boîte courrier 187, 75252 PARIS CEDEX 05, France

pa@raviart.com 